The International Journal of Engineering and Science (IJES)

|| Volume || 6 || Issue || 5 || Pages || PP 57-87 || 2017 ||

ISSN (e): $2319-1813$ ISSN (p): $2319-1805$

\title{
Short Review of the Unitary Quantum Theory
}

\author{
Leo G. Sapogin ${ }^{1}$, V. A. Dzhanibekov ${ }^{2}$, Yu. A. Ryabov ${ }^{3}$ \\ ${ }^{1}$ Department of Physics, Technical University (MADI), 64 Leningradsky pr., A-319, Moscow, 125319, Russia, \\ ${ }^{2}$ Department of Cosmophysics, Tomsk State University, 36 Lenina st., 634050, Tomsk, Russia. \\ ${ }^{3}$ Department of Mathematics, Technical University (MADI), 64 Leningradsky pr., A-319, 125319, Moscow,
}

Russia

\begin{abstract}
The paper proposes a model of a unitary quantum field theory where the particle is represented as a wave packet. The frequency dispersion equation is chosen so that the packet periodically appears and disappears without changing its form. The envelope of the process is identified with a conventional wave function. Equation of such a field is nonlinear and relativistically invariant. With proper adjustments, they are reduced to Dirac, Schroedinger and Hamilton-Jacobi equations. A number of new experimental effects are predicted both for high and low energies.
\end{abstract}

Keywords: Unitary Quantum Theory, Standard Model, Quantum Electrodynamics, Maxwell Equations, Schrödinger Equation, Solid State.

Date of Submission: 14 May 2017

Date of Accepted: 30 May 2017

It is difficult, if not impossible; to avoid the conclusion

that only mathematical description expresses all our

knowledge about the various aspects of our reality.

- An opinion extracted from a Soviet newspaper

\section{INTRODUCTION}

It seems that the majority of researches have absolutely forgotten the fact that one of the master spirits of contemporary world, A. Einstein, till the end of his life had not adopted the standard quantum mechanics at all. Better to cite his well-known words: «Great initial success of the quantum theory could not make me believe in a dice game being the basis of it. I do not believe this principal conception being an appropriate foundation for physics as a whole... Physicists think me an old fool, but I am convinced that the future development of physics will go in another direction than heretofore I reject the main idea of modern statistical quantum theory... I' $m$ quite sure that the existing statistical character of modern quantum theory should be ascribed to the fact that that theory operates with incomplete descriptions of physical systems only.» A.Einstein (back translation).

At the first stage of quantum mechanics evolution in the frame of classical physics theory the mechanism of corpuscular-wave dualism was not discovered at all, as it was done later in the UQT [2-4, 13-15, 23]. It is worth a surprise that the super abstract quantum ideology ad hoc designed by Niels Bohr was suitable in general for the description of quantum reality. An explorer did contradict anything by strictly using new frequently paradoxical quantum rules, and any paradox could be removed by the simple prohibition of its analysis. Although many researches tried to solve these problems they were not successful. The outspoken interpretation of quantum theory had become out of any criticism. More over the determination of simulators describing one of the sides of quantum reality had been announced as the main target of quantum science, while the picture in figures and a-going had become simply an optional target.

Nevertheless one general philosophic problem had been remaining: the dual principles of the fundamental physics. There were particles as some points being the source of a field that could not be reduced to the field itself; the researchers did not do their utmost, though. Introduction of this micro-particle had resulted in a wide range of different divergences - anybody knows that electric power of a point charge equals infinity. A lot of ideas had appeared, absolutely brilliant ideas from mathematical point of view, suitable for these appearing infinities abolishing. We can use as a cover the words of P.A.Dirac: "most physicists are completely satisfied with the existing situation. They consider relativistic quantum field theory and electrodynamics to be quite perfect theories and it is not necessary to be anxious about the situation. I should say that I do not like that at all, because according to such perfect theory we have to neglect, without any reason, infinities that appear in the equations. It is just mathematical nonsense. Usually in mathematics the value can be rejected only in the case it were too small, but not because it is infinitely big and someone would like to get rid of it". Direction in 
Physics, New York, 1978 (back translation).

The substantial success of the quantum mechanics (particularly in the stationary cases) was based on the simple correlation of de Broglie wave length and geometric properties of potential. Formally the particle was considered as a point; in other case it was difficult to add probability amplitude character to the wave function. But the point-character of a charge as well the principle of Complementarity did not allow to go ahead in the elementary particles structure and thus the further development of the quantum theory of the field in the frames of the assumed paradigm had resulted in total fiasco of the field quantum theory itself.

There is another concept in physics; it comes from W.Clifford, A. Einstein, E.Schrödinger and Louis de Broglie in which the particle is considered as a bunch (wave packet) of a certain unified field. The position of associates of the concept would be expressed the most clearly by the following words of A.Einstein: "We could therefore regard matter as being constituted by the regions of space in which the field is extremely strong. A thrown stone is, from this point of view, a changing field in which the states of the greatest field intensity travel through space with the velocity of the stone. There is no place in this new kind of physics both for the field and the matter, for the field is the only reality... and the laws of motion would automatically follow from the laws of field". (back translation). By (M. Jemmer, [1]) definition of the particle as a wave packet is the item for some unitary theory. The first articles concerning this matter were published in [2-4]. The entire term "unitary" belongs to who has classified quantum wave's theories, and it is correlated with the theories that represent particle as a wave packet [1]. In Unitary Quantum Theory a particle is described as a wave packet that in its movement is periodically spreading along the Metagalaxy and is gathering again. For such moving wave packet both the relativistic and the classical mechanics follow from these unitary quantum equations, probably the Maxwell equations and the gravitation follow from exact UQT equations [8, 13-15], but this has not been proved yet being the problem of the future. Nevertheless the UQT scalar equation (a telegraph type) in general makes it possible to obtain not only Schrödinger but also Maxwell equations [14, 15].

The field of investigations of the Unified Unitary Quantum Theory (UUQT) is the most profound level of substance: the level of elementary particles and quantum effects.

As well known all particles have besides corpuscular properties wave properties too (particles can interfere with each other or with themselves), and their behavior is described by means of the wave function. In the case of a particle moved in the free space, the wave function is described as de Broglie plane wave which wavelength is inverse to the momentum of the particle. If the particle is slowing down or accelerating by applied fields then its wavelength is increasing or decreasing, respectively. The wave itself has no physical interpretation, but the squared value of its amplitude is proportional to the probability to find the particle in a defined place. That is why these waves are also called "waves of probability" or "waves of knowledge", etc.

There is another problem: the particle has no exact value for coordinate and for momentum at the same time, although either value could be measured arbitrarily closely (uncertainty relation).That is why the definition of trajectory of a quantum particle has no sense.

As opposed to the laws of the classical physics with its determinism where one can predict results of the motion of separate particles, in the quantum theory one can only predict the probability of the behavior of separate particles. Even the nature does not know the way a particle goes by in the case of diffraction by two slits. But it is not the most depressing. The Quantum Physics has wave-corpuscle dualism as well as field dualism and matter dualism. All particles act as sources of field, but it appears that they are only points which have no relation to these fields, and one can't tell anything in concrete about them.

Let us continue to confuse the reader. We shall consider an extremely simple experiment with single particles in the terms of the modern quantum theory. It will allow us to understand what is going on and will be useful for us in the future. Let single photons fall on a semitransparent mirror directed at the angle of 45 degrees to their stream. Semitransparent means that a half of the falling light is reflected and another one passes by. Photon counters are installed on the paths of reflected and passed rays (Fig.1).

Particies

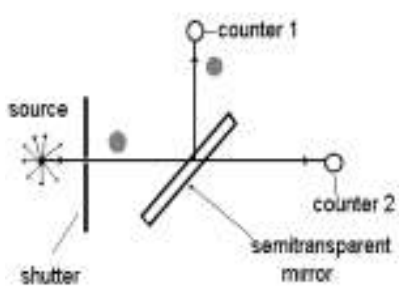

Waves

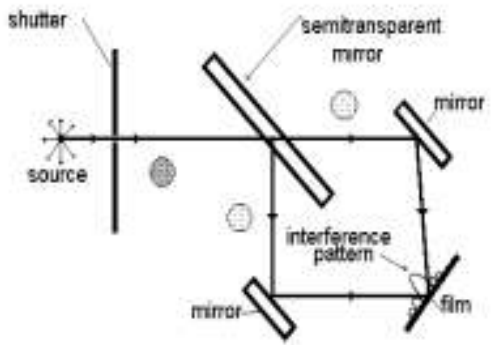

Fig.1. Experiments with individual photons on semitransparent mirror. 
In the terms of the wave theory everything is simple: an incident wave will be reflected and will be passed partially.

But particles as they are indivisible have to be reflected or be passed by. If a counter of reflected beams particles registers an event it is evidently to suppose that the second counter will register nothing. It is easy to see that if one will re-unite passed and reflected beams and sends them to the screen then...it's all about the way how we are going to argue.

From the wave theory there will be an interference pattern, but from the corpuscular theory it will not occur. In fact, an interference pattern is observed in experiments even for single photons, and our suppositions are wrong to say the least. In order to spare the doubts about how is it possible, it is better to forbid one to think about it. And the principle of Complementarity in modern physics does it in any case.

It allows to ask only the questions for which it's possible to give an answer by experimentally only. When one tries to find a particle it means that one rejects to observe the interference pattern and vice versa. As though we could know from experiment either a particle has passed by or has been reflected, we would realize the real particle behavior. But it`s impossible to do by the means of macro-instruments.

The principle of Complementary makes the quantum physics descriptively inaccessible."There are many experiments, that we just cannot explain without considering the wave function as a wave that influences on the whole region and not as particles appearing may be here, may be there, as it is possible in the terms of the clearly probabilistic point of view"(E.Schrödinger). In other words a wave acts in the whole area simultaneously, not "may be here, may be there", otherwise there wouldn't be any diffraction or interference.

Eventually we have to admit that the prohibitions of the principle of Complementarity respond to the weakness philosophy, and the role of this principle is obviously analogous to the role of a calorie, a phlogiston and other obsolete concepts.

\section{THE UNIFIED FIELD THEORY APPROACH}

Let us ask the questions that are forbidden by the principle of Complementarity. What is the wave of an electron? What is the behavior of an electron indeed, when nobody looks at it? (it's natural behavior?) How does it manage to go through a potential barrier when its energy is less than the barrier height (tunneling effect)? How does it, as it is indivisible, go simultaneously by two slits which are divided by a great distance in comparison with its own size? What kind of structure has an atom of hydrogen constructed at the lowest energy state ( $s$-state)? How can the probabilistic consideration of a wave function to result from the mathematical formalism of the theory? Why is the actual Quantum Mechanics reversible? This is a primary law, and the irreversibility has to follow from it for dispose the paradoxes in the statistical mechanics. Last but not least: what structure has the electron itself described in the terms of probability? This is a huge complex of mysteries. All (or almost all) physicists resigned and even prefer not to speak about it. But there is also someone who does speak. Paul Langevin even called the formalism of Quantum Mechanics with its principle of Complementarity the "intellectual debauch".

E. Schrödinger wrote that he "was happy for three months" when he had got the idea to consider the particle as the packet (bunch) of de Broglie waves until the English mathematician Darwin proved that the packet would spread and vanish. But the trouble of all of these attempts (E.Schrödinger, Louis de Broglie, etc) was the fact they always tried to construct it by means of de Broglie waves with such dispersion that any wave packet has to spread. The including of nonlinearity (Louis de Broglie) just extremely complicated the problem but didn't solve it.

\section{THE UNIFIED UNITARY QUANTUM THEORY INTERPRETATION}

Ernst Mach's outlook is well characterized by an episode from his life. Mach was studying ballistics and was often presented on the shooting grounds. Once he said to a colleague: "There is a question, which is constantly torturing me: Does the shell exist in the interval between the shooting and the hitting of the target? We do not see or feel it in any way." "You are crazy," his colleague answered; "How can you doubt the existence of the shell? You yourself are calculating its trajectory, and your calculations agree with the experiment. Is this not proof of the shell's existence?" "It does not prove anything," Mach objected. "The trajectory might only be a supplementary mathematical notion serving to predict further observations. The shell might not be moving along the trajectory at all. It might disappear at the moment of the shooting and reappear again at the moment it hits the target." The colleague only shrugged his shoulders in surprise. But Mach did not stop there. In order to solve this problem he designed a special device for photographing the shell in flight. Mach was not only convinced that the shell existed in flight, but he also saw on the photos certain lines coming from the shell, which were called Mach lines.It was due to his doubts about the existence of an unobserved flying shell that Mach created the supersonic gas dynamic theory. As a tribute to his achievements, the ratio of a flying object's speed to the speed of sound is called the Mach number. 
H. Laitko and D. Hoffman, Matters of Natural and Technical History, $1988\left(4^{\text {th }}\right)$, pp. 45-57.

The critical feature of the Unified Unitary Quantum Theory (UUQT) is the fact that it describes the particle as a bunch (packet) of certain unified field, but not as a questionable structure of the de Broglie waves of probability. For spying upon the particles which we consider as very small bunches of the real field, let us consider a Hypothetic Observer (HO) which is able to measure the parameters of these bunches with the hypothetic microprobe. Dimensions of microprobe are much less than the dimensions of the particles. The result of these measurements will be certain structure function that describes bunch of the real field. Obviously, this hypothetic $\mathrm{HO}$ and microprobe couldn't exist, but our thought experiments will be as simple as possible.

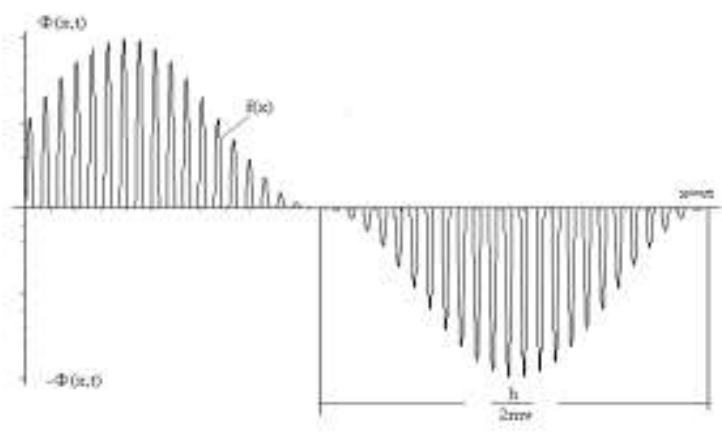

Fig. 2. Behavior of wave packet in linear dispersion medium (i.e., rather like a series of stroboscopic photographs).

If we choose the dispersion of these partial waves equal to linear, we could have an extremely curious process, which mathematical formulation used never before. If we have dispersion, then harmonic components of partial waves propagated with different velocities will result in spreading of the wave packet over all space or over all Metagalaxy. Mathematical investigations show that the spreading goes on without any changes of the form of the wave packet; but at the end, there is a moment when a wave packet vanishes at all. Where does its energy disappear to? It remains in the form of harmonic components that set up a certain background in any point in the space. As these waves are not damped and continue to propagate with velocity of their own, then after a while the wave packet begins to revive in another point, but its sign will be changed at that. During the motion, the packet will appear and disappear periodically (Fig. 2).

The envelope of this process is locus of points, locus of points of its maximum, it is a sinusoidal quantity and it rests in all reference frames; in other words, its phase velocity is equal zero in any reference frame, i.e. it's relativistically invariant (only by means of it the results of the relativistic dynamics are absolutely correct). If we change a reference frame, we will receive a different value of wavelength of the envelope, but it will be motionless as well. As the computing shows the wavelength of the envelope is exactly equal to de Broglie wavelength, and the dependence of this wavelength on packet velocity is the same! As you see, all the Unified Unitary Quantum Theory is occupied with the resolute exploiting of this basic idea. It should be stressed that this periodical appearing and disappearing of particles doesn't refer to the Quantum Mechanics, as an immovable packet doesn't oscillate. The requirement of the relativistic invariance, that would be the main requirement for any theory, specifies the idea further. It states the following: when Lord has excited in space continuum wave packet with his finger and then he has taken it away, then the packet will go on oscillating as a membrane or a string after impact. The frequency $\omega_{S}$ of these free oscillations is very high: it is proportional to the rest energy of the particle and it is equal to the frequency of the so called Schrödinger`s trembling (“zitter-bewegung").

$$
\omega_{S}=\frac{m c^{2}}{\hbar \gamma}, \quad \gamma=\sqrt{1-v^{2} / c^{2}}
$$

Within the motion there arise de Broglie vibrations with frequency $\omega_{B}=m v^{2} / \hbar \gamma$ due to dispersion. At small energies $\omega_{S} \gg \omega_{B}$ and the presence of quick own oscillations has no influence on experiment. So, all quantum phenomena result from de Broglie oscillations.

The value of frequency $\omega_{B}$ tends to $\omega_{S}$ with growth of energy and resonance phenomenon appears that results in oscillating amplitude increase and in mass growth. Thus the well-known graph of particle mass dependence on the velocity (Fig.3) approaching to lights velocity constitutes actually a half of usual 
resonance curve for forced oscillation of harmonic oscillator if energy dissipation is absent. In the case when $\quad v \rightarrow c$, frequency $\omega_{B} \rightarrow \omega_{S}$ (frequency resonance), $\gamma \rightarrow 0$ and the beats appear with difference frequency

$$
\omega_{d}=\omega_{s}-\omega_{B} \approx m c^{2} \gamma / \hbar
$$

and particle will obtain absolutely new low-frequency envelop with wave length

$$
\wedge=\frac{h}{m c \gamma}
$$

This is a new wave. This can be checked experimental in CERN. It would be perfect to examine by experiments at future accelerators the appearance of such new wave with the length growing together with energy [2-4, 17-19]. In ultra-relativistic limit case the value of $\wedge$ becomes much greater as typical dimension of quantum system it (new wave) interacts with. Now the length of new wave grows with energy contrary to de Broglie wave length slowly decreasing, and particle requires the form of quasi-stationary wave packet moving in accordance with classical laws. That explains the success of hydrodynamics fluid theory concerning with numerous particle birth when the packet having extremely big amplitude is able to split into series of packets with smaller amplitudes.

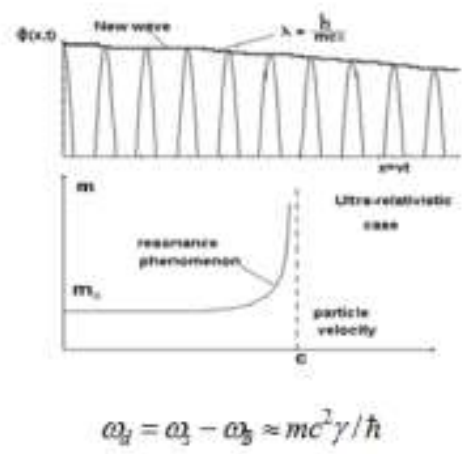

Fig. 3 .

But such splitting processes characterize not only high-energy particles. Something like this takes place at small energies also, but overwhelming majority of arising wave packets are under the barrier and so will not be detected. For example see also the Fission of Elementary Particles and the Evidence for the Fractional Electrons in Liquid Helium [63]. In a cover [64] about it there is next: "One man think that the electron split. If he's right it's curtains for quantum theory".

It would be perfect to examine by experiments at future accelerators the appearance of such new wave with the length growing together with energy [2-4, 17-19].

If our $\mathrm{HO}$ places at the way of motion of the wave packet quite a number of his microprobes, then due to the dispersion spreading and rebuilding he can observe the envelope of this process, and all of this will not be at variance to the general Quantum Mechanics, as this envelope corresponds to the wave function. This figure, i.e. a sinusoidal envelope with a regular shape, can be seen by the $\mathrm{HO}$ in the only case: if the only single particle would exist in the world. But the real world consists of an enormous number of particles moving each other with different velocities. The partial waves (harmonic components) of those particles which have vanished at this moment can be summarized and emerge real fluctuations of the field or in other words the vacuum fluctuations that will act in a random manner.

These fluctuations could destroy all idyllic character of measurements of our HO (Hypothetic Observer) for single particle in Universe because the sinusoidal envelope will be distorted by vacuum fluctuations and it will be difficult to separate it clearly. Any wave packet that is described in the terms of the becoming structural function could be decomposed by means of Fourier transforming into plane sinusoidal (partial) waves.

These waves are infinitely numerous, and their amplitude is infinitesimal. If we summarize them it will emerge zero everywhere except of the area occupied by the structure function. Thus the structure function could be represented either as a function of time (time representation) or as a function of an amplitude of harmonic components related to frequency (spectral representation). It is absolutely equivalent to mathematical representations.

Now there is no necessity in the principle Complementary that was a very convenient view ad hoc. It is easy and clear how the synthesis of corpuscular and wave properties is realized. Corpuscular properties occur due to 
the localization of a wave packet in a small spatial region. The wave properties of the de Broglie waves can be explained in the following way: when the wave packet approaches to the diffraction system (for example Young's experiment with two slits) then we have an ordinary diffraction of partial waves by splits, and the diffraction pattern of partial waves appears at the screen. $\mathrm{HO}$ could observe it with his microprobes.

As these packets are not overlapped then everything is linear and the superposition of the partial waves creates a total diffraction pattern modulated by the de Broglie wave, although the plain de Broglie wave doesn't exist at all. It should be stressed that de Broglie wave is a packets locus of points of maximum in his motion, and it is a superposition of partial waves, that is why it appears in any diffraction and interference experiment.

\section{THE QUANTUM MEASUREMENTS}

Let us try to consider real instruments, which are always macroscopic. Atomic nuclei and electron shells are situated very near to each other and form a very numerous, but discrete series. A transition from the one such a state to another is a quantum jump. That is why the absorption and emitting of energy between the atomic systems is carried out by means of the quanta. However, it doesn't mean that in the motion process the quantum or the particle propagates as something constant and indivisible. The energy of the particle can be divided or changed by vacuum fluctuations. The wave packet of a photon, for example, can, in the issue of the overlapping of vacuum fluctuation, turn into meson at short time, and photon can disguise oneself as a proton or as a neutron. It $\mathrm{s}$ assumed in the ordinary quantum field theory that a proton has an atmosphere mesons; it follows from the interpretation of the results of its collisions with another particles. There is no mesons atmosphere indeed. A proton appears and disappears during its motion constantly at the de Broglie wavelength, and its mass changes periodically from the double value of a proton's mass to zero, taken on the intermediate values of mesons masses.

Eventually, all of the quantum measurements are based on energy absorption and present inconvertible processes [5, 6, 13-15]. For every instrument founded a particle will operate, a quantum of energy is needed at least, thus it is a threshold energy of instrument defining it's responsively. By the way, we would like to notice that our HO (Hypothetic Observer) uses the instruments with zero threshold energy that is why it can register even vacuum fluctuations.

Let us consider the process of interaction of a particle with a macro-instrument [5, 6]. As soon as the particle is a wave packet, its energy is proportional to the intensity of the packet, but it can be changed because of periodic spreading's and appearances. Besides the packet itself can be divided during the interactions. The macroinstrument to register a particle has to wait for a moment when the total energy of the particle and of the fluctuation of the atom would be more or equal to threshold energy. It is clear that the probability of the operation of the apparatus will be proportional to the amplitude of the wave packet, or more exactly, to the value of intensity of the envelope of the wave function. If the wave packet with a too low intensity in comparison with threshold energy of the macro-instrument approaches to the macro-instrument, the great fluctuation of vacuum is required, but the probability of such an event is too small, and it means that the probability to detect the particle is small too (Fig. 4). The theory of the quantum measurements is developed in the Unified Unitary Quantum Theory (UUQT), and the statistical interpretation follows now from the theory, but not just postulated, as it was before in the conventional quantum theory. This point of view requires automatically that the value of the dispersion of vacuum fluctuations is finite that, in another turn, requires the finiteness of the Universe.

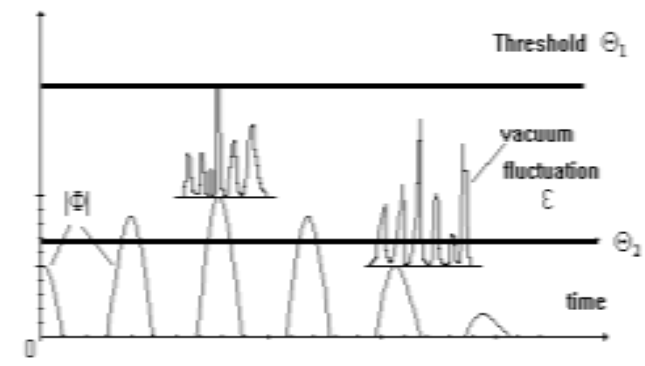

Fig.4. Quantum measurements.

\section{THE UNITARY QUANTUM ILLUSTRATIONS}

The uncertainty relation arises because energy and momentum are not constants, but they periodically change because of the dispersion owing to disappearance and appearance of the particle [2-4]. Besides because of statistical laws of measurements with macro instruments, there is no any way to measure anything accurately owing to the unpredictable fluctuations of the vacuum. HO (Hypothetic Observer) could predict the coordinate, 
the momentum or the energy of the packet, if he would be the only one in the Universe, i.e. in the case of absence of the vacuum fluctuations.

The presence of unpredictable vacuum fluctuations makes all of the laws of the micro-world principally statistical for any observer. An accurate prediction of expected events requires an accurate knowledge of the vacuum fluctuation in any moment of time, what is impossible, because it is necessary to have the information on the structure and the behavior of any packet (particle) in the universe and to control their motion. The mechanical determinism of Laplace [42] went absolutely lost in the modern physics as well as in the future one. Maxwell was right when he told; "the true logic of the universe is calculation of the probabilities". (back translation). The envelope of partial waves, occurring due to linear transformations at the wave packet and being in the ruins of splitting of the packet corresponds to Huygens principle. It explains how the relating of a moving particle with a monochromatic de Broglie wave is formally possible, propagating in the direction of the motion, and with all wave properties. There are partial waves that we consider as participants of diffraction and interference, but due to the principle of superposition we get the same result as if it a de Broglie wave would participate at the process.

The new linear equations of the UUQT allow the time inversion with simultaneous replacing of the wave function with a conjugated one, with the formal reversibility. Actually this reversibility takes place just in the case if the Universe consisted of the only one particle, as in the real world the recovering of the previous vacuum fluctuation is also needed for the total reversibility of the process. But there is a simultaneous reversibility of all processes in the Universe required for it that is impossible. It doesn 't mean that quantum processes are inconvertible, just the reversibility has a statistical character, but now direction of the current of time defines entropy only.

The envelope, introduced before, is accurately monochromatic, but it does not exist as a traveling plane wave with such properties in the reality. Though it is related to the energy of the particle, the following definitions, such as "waves of the probability", "waves of the knowledge", could be related with it too. In contrast to the general quantum theory, now a very important phase is coming. It is the most easy to show it as the tunneling effect.

We would like to mention these established quantum phenomena to the reader. If we have a sufficiently narrow barrier with the height that is larger than the energy of an incident particle, according to the classical mechanics it will never go through the barrier. In the general quantum theory, the incident wave reflects and passes by partially, and we have a finite quantity of the probability that the particle will be behind the barrier. In these cases the general Quantum Mechanics states that the particle makes a tunnel in the barrier for itself, hiding the method of creation of this tunnel.

Let us listen to what HO says of this process? If a particle is approaching closely to a potential barrier in the phase of an absolute collapse, then it easily goes through the barrier, not interacting with it because of linear of all of equations for the small amplitude of the field. It just appears behind the barrier, without interacting with it, if its width is much less than de Broglie wavelength. And there is no necessity for it to make a tunnel. However, if it approaches in the phase with the maximal value of the packet, then the particle would be reflected because of the nonlinear interaction of the waves with the field of the barrier.

Now let us return to the experiment with the semitransparent mirror, discussed above. In terms of the described point of view, the wave packet (particle) will be divided at the mirror and enter in every beam, that depends on the packet phase near the mirror and on the structure of the mirror in this place. We have, in general, two not equal wave packets fragments with less values of the amplitude that can interfere. The changing of the parts of the fragments does not follow by because all process are linear, i.e. they are not dependants on amplitude. Besides the probability of detecting of the fragments is reduced, because an appreciable fluctuation of the vacuum is necessary for arising of threshold of detection of the counter. Consequently, in the results of the measurements the particles have to be lost or be observed as single particles in both of the beams simultaneously. The creation of two particles from a single is not a confusing fact, because the energy of the fragments will be reconstructed to the necessary level by means of the vacuum fluctuation. Note, the statement of Standard Quantum Mechanics that the particle may be presented simultaneously in many points of quantum world sounds strange from the common sense and remained for decades without any understanding of principal things. Within bounds of UQT scientific explanations are correct in principle $[59,60]$.

At present time we have an ambiguous situation when high-tech experiments with fantastic results have been carried out, for example the classical experiments of Brown and Twiss and the variations of them (Fig. 1). It was found out that frequently both of the counters detect particles simultaneously, that is confirmed by the proposed mechanism. Furthermore, most of such experiments (including experiments with entangled photons) confirm directly this interpretation. The results of experiments with entangled particles are quite simple and understandable within bounds of UQT, and the idea to seek some over light mystic relations between particles is fully meaningless. 
In consequence, an increasing number of photon pairs is always observed in the beam of light. However, it was found out that it is possible to carry out experiments whose effect remains also in the situation when there is no any way for any induced radiation. If we will collide particles of any kind, and if in the colliding point one or two particles are vanished, then they have to go against another without any interaction. Indeed, in the protonproton interactions $6 \%$ of the particles don't interact, but go through the others. An analogous effect takes place in the atom of hydrogen in the state of minimum of energy. It is well known that this $s$-state is not rotational, and Bohr-Sommerfeld's atom model describes the spectrum strictly in the relativistic case. If we apply this model to the $s$-state of the electron, we will obtain that the paths of the electron pass through the nuclear, and they were early excepted as absolutely absurd. Today it is clear that an electron just oscillates along a straight, going through the proton. All this allowed one of the authors to consider the problem of deuteron-deuteron interaction in other respects and to predict the cold fusion [7, 11].

Quantum object is getting classical one with a simultaneous increasing of its mass, i.e. in the case of superposition of a large number of wave packets. The case when all of packets consisting a body will consolidate and spread simultaneously is impossible in physics, as they have different velocities and masses. That is why such a combination seems as a stable and permanent object, moving according to the classical mechanics laws, though every packet is described in terms of the Quantum Mechanics. It looks like all particles in the Universe owe their existence to each other, and the Universe itself is just a mathematical illusion, a trick. Remember "The Tempest" of William Shakespeare:

We are such stuff

As dreams are made on;

And our little life

Is rounded with a sleep.

In justice to the adherents of the Complementary we have to say the following. They do not retract it, though they have to wriggle, they have to tell that particles always go to the mirror as correlated pairs, and one of them goes through, but the second is reflected. Of course we need to consider the induced radiation effect, when the one atom's radiation is increasing the probability of emitting from another excited atom of the same source, but it does not always happen. Let us return to the principle of Complementary. It is clear, that if we would not be interested in the nature of the particle and consider it just as an indivisible point then the principle of Complementary is correct. It is a very curious principle and it is amazing how N. Bohr could invent it.

In recent years a numerous of experiments was carried out, which found out superluminal speeds. Not debating if the special theory of relativity is right or not, let us show that in the Unified Unitary Quantum Theory (UUQT) any velocity is possible and the velocity of light is not maximum possible.

Let us consider Euclidean plain space, in which the photon propagates along the $X$-axis. According to the UUQT it is a wave packet and it could be presented as an infinite sum of harmonic components, that exist on the $X$-axis, figuratively speaking, placed at a distance of a million light years ahead and backwards. Now if we place on the $X$-axis arbitrarily far some special device, creating an anomalistic high dispersion, then the photon could occur at the exit of the device, because the harmonic components shifted each other. The most interesting in this process is that nothing has moved between incident and reconstructed photons at this velocity! With other words, the conventional definition of the velocity is getting obsolete [19, 23].

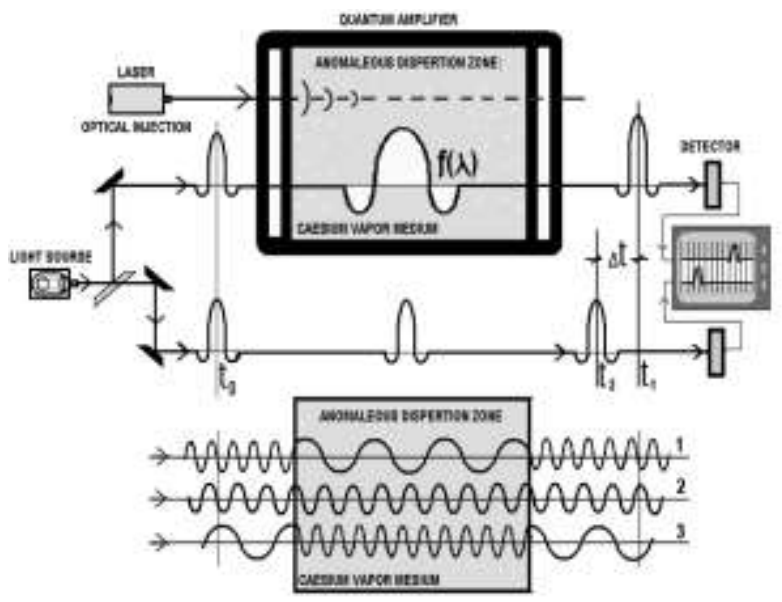

Fig. 5. Experiments of L.Wang - superluminal light propagation.

Such experiments were carried out by several teams (in Berkeley, Vienna, Cologne, Florence, etc.) and they 
emerged the superluminal speeds. The most interesting were Lijun Wang`s investigations [27] in which the velocity 310 times higher than the speed of the light (Fig. 5) was found. Wang gives the same interpretation as ours, but only for an impulse of light. In this case it is a wrong interpretation, because in the experiment the envelope of the light pulse is not distorted absolutely, but it has to be obligatory, and Wang notices that it is amazed. He supposes that the special theory of relativity is absolutely destroyed. But it is not quite true.

Our idea that particles are wave packets is an absolutely original idea for the world wide science. The waves at the Fig. 5 have to be realized as separated partial waves of the spectral decomposition of the wave packets of the separated photons, but not as a spectral decomposition of the light pulse. Then the form of the momentum envelope will not be distorted.

The aspects of the Unitary Quantum Theory are confirmed by results of their practical applications to traditional tasks of physics. The UUQT allows firstly in the international science, not either to compute the electron charge and the fine structure constant $\mathbf{( 1 / 1 3 7 )}$ with the great precision $(\mathbf{0 . 3 \%})$ [9-10, 61]. Some late the Unitary Quantum Theory allows computing the mass spectrum of all elementary particles without any adjusting parameters $[15,16,18]$. By the way computed spectrum has particle with mass=131.51711 $\mathrm{GeV}(\mathrm{L}=2, \mathrm{~m}=2)$. Once desired it can be called ""Higgs boson"", it lies within declared by the CERN+Tevatron mass interval 125 $140 \mathrm{GeV}$ expected to contain ""Higgs boson"". CERN promised to obtain more precise mass value by December 2014. Some late were find 3 pentaquarks. The significance of each of these masses is more than 9 standard deviations. One has a mass of $4380 \pm 8 \pm 29 \mathrm{MeV}$ and a width of $205 \pm 18 \pm 86 \mathrm{MeV}$ (our theory mass=4315, $87 \mathrm{MeV}(\mathrm{L}=9, \mathrm{~m}=0))$ while the second is narrower, with a mass of $4449.8 \pm 1.7 \pm 2.5 \mathrm{MeV}$ and a width of $39 \pm 5 \pm 19 \mathrm{MeV}$ (our theory mass=4496,65 MeV (L=7,m=2)), third $\Theta+$ barion has mass=1522 $\pm 3 \mathrm{MeV}$ (our theory mass=1524.62 MeV $(\mathrm{L}=12, \mathrm{~m}=0)$ ). Report number: CERN-PH-EP-2015-153, LHCb-PAPER-2015029. All this masses were calculated in 2007 [15, 16, 18, 56, and 58]!

The Modern Standard Model and quantum theories of field couldn't even raise these problems mathematically. It should be stressed than when we will find the spectrum of masses and charge of electron, time won't be a part of the ultimate equations and it will stay Newtonian. In the Unified Unitary Quantum Theory all interactions and particle production (packet split) are considered as an effect of diffraction of the packets by each other because of the nonlinearity. An analytical solution of these tasks will require new mathematical methods, and it is not even clear how to start with it at presence.

\section{THE APPROXIMATED EQUATION WITH THE OSCILLATING CHARGE}

There are strong hard rules in the modern theoretical physics. Any new theory has to include classical results. This is strictly satisfied because the Hamilton-Jacobi relativistic equation and Dirac equation follow from the UUQT, i.e. all modern basics of the fundamental quantum science. In the linear equations of the UUQT the mass was replaced by the rest energy divided to square speed of light, and then the system of 32 nonlinear integro-differential equations appears as a consequence. They were firstly found out by L. Sapogin and V. Boichenko [8] in 1984, and only in 1988 they solved the dimensionless scalar version of this equation that allows to get the fine structure constant $1 / 137$ and electron charge with accuracy $0.3 \%[9,10]$.

In this approximation of the UUQT, the wave packet is realized as a spatial divided electric charge that oscillates, its equation depends on time, coordinate and velocity and it could work in the rough model of the particle as oscillated charge, so we can exploit the Newton equations. It is becoming easy to see the tunneling effect: while the moving particle is approaching to the potential barrier, in the phase when the charge is extremely small, it is easy for it to go through the barrier, and when the quantity of the charge is large, the repulsion force is increasing, and the particle will be reflected.

The numerical solution of these equations [14-16, 20], for the most common quantum tasks emerges approximately the same results as the calculation of the general Quantum Mechanics (QM). By the way, by means of the UUQT it is possible to get this equation from the Schrödinger's one with very low energies [14, 15]. But there are though some interesting differences. The equations of motion of the oscillated charge were not treated in physics before and they have an important difference from the classical laws of motion - the invariance of the motion in the relation to invariance translations. It means the absence of the great classical momentum and energy conservation laws. They appear in the UUQT and then in the classical mechanics only with an averaging for all particles.

\section{UNCERTAINTY RELATIONS}

Now we obtain Uncertainty relations $[13-15,19,23]$. As far as the particle (wave packet) is periodically appearing and vanishing at de Broglie wave length (more precisely, the packet disappears twice, and the probability of its detecting is sufficiently big in maximum region only) the position of such a packet may be detected with error 


$$
\Delta x \geq \frac{\lambda}{2} \text { and then } \quad \Delta x \cdot P \geq \frac{h}{2}
$$

As at measuring of momentum module is inevitable the error $\Delta P={ }^{*}$, then we have following inequality:

$$
\Delta x \cdot \Delta P \geq h \text {. }
$$

The statements of standard quantum mechanics that particles do not have a trajectory become more understandable. Of course, there is a lot of truth in those words. First, it is possible to say so about intermittent (dotted) motion of the particle with oscillating charge. Second, any packet (particle) is able during its motion to split into few parts. Each of those parts being summed with vacuum fluctuation may product, in principle, some new particles. Or visa versa the broken particle may vanish at all and contribute to general fluctuating chaos of the vacuum. But in any case it is better to have more clear idea of particle concrete motion than operate with generally accepted nowadays-obscure sentence about lack of trajectory.

\section{THE HARMONIC OSCILLATOR IN UQT}

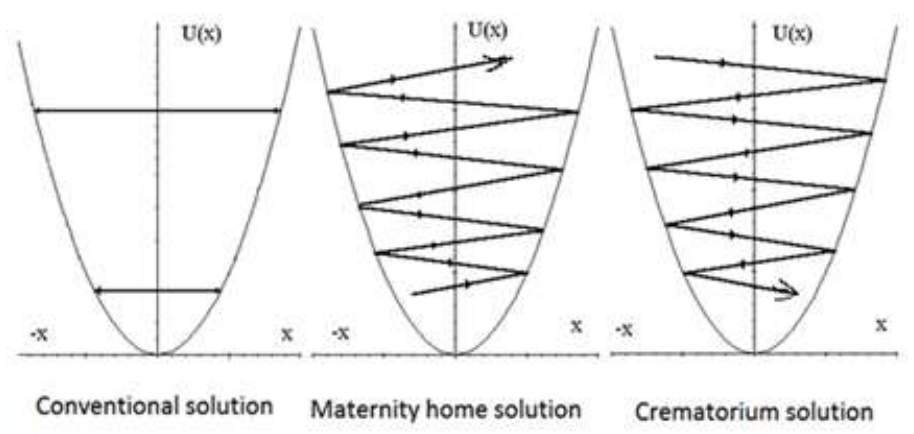

Fig. 6.

The consideration of the problems concerning oscillations of particles with an oscillating charge in a parabolic well (harmonic oscillator) besides the common results of QM for stationary states results in two different solutions that are shown on Fig. 6. New amazing solutions appeared, one of them was called "Maternity home" and another was called "Crematorium". In the first case the energy of the particle can increase indefinitely, furthermore if we proceed from a very low initial quantity in the equation, it results in the increasing of the energy of the particle in the production of the matter, indeed. The second solution could due to collapse (disappear) of the matter-particle. These solutions are logically independent directly, and their appearance depends on initial phase. With other words, one solution describes the matter (energy) production, and another one its collapse; and it may be said that the Unified Unitary Quantum Theory (UUQT) allows describing the creation of the matter and the Universe, but not as a result of the Big Bang. The Universe wouldn't be given to us in the static form, it arose in some way and it continues to develop, and we could see that one of the basic features is the filling of space by matter.

\section{THE NEW SOURCES OF ENERGY}

As well known, in all experiments the local law of energy conservation (LEC) and the law of conservation of momentum in individual quantum processes are correct only for high-energy states. For low energies we can't claim that, because of the uncertainty relation and the stochastic nature of QM`s predictions. That is why the idea of the global, but not of local LEC exists invisibly in the QM and it is not a new one.

For the physics it only means that for the stationary solution with fixed discrete energy levels (the general QM) of the velocity of the particle reflected by a wall is equal to incident one. The UUQT allows to consider another ways too. Thus if the velocity of the particle for every reflection is decreasing, then it is corresponding to the "Crematorium" solution, but if it is increasing, then it is corresponding to the "Maternity home". What scenario would turn to the reality depends on the initial phase of the wave function and on the energy of the particle. Besides the UUQT is fundamentally inapplicable for closed systems, because such systems are idealizations, which are very useful, but not according to the base of consideration used in the UUQT.

Anyway, the whole modern science, including the Quantum Mechanics (QM), is still based on the great LEC. However, there is a difficult situation in the Quantum Mechanics. It deals with the fact that the LEC follows only from the Newton mechanics. QM generalizes the facts of the classical mechanics including all of its laws, but its results have a sufficiently statistical nature, they are correct only for large amounts of particles. But how 
do we have to consider single particles, with their individual processes? It appears that for the single particles LEC does not follow from QM (!), thus individual events are absolutely incidental and do not follow this law. To evade this question it was announced that Quantum Mechanics does not describe individual events (!?)

Let us discuss a thought experiment. To make our reasons more simple let operate a certain quantum ballparticle. If the ball is approaching to the wall, then its velocity after reflection will always be equal to the incident velocity (here we neglect a quantity of the friction force and consider that the ball and the wall are perfectly elastic). In the case of the quantum ball the velocity after the reflection would possess the whole arrange of the values, in different experiments under equal conditions. There would be some balls that would be reflected with velocities that are higher and some that are lower than the initial velocity, and some of them with velocities equal to the incident one, and every case would be considered statistically in the terms of the Quantum Mechanics.

Let us answer the following question: what would happen if we place another wall opposite the first, and would try to increase the velocity of the ball after every reflection? Then we would get increasing of energy of the ball without action of any external force. The energetic of the systems in the XXI century will treat the question of constructing of initial conditions for a numerous quantity of particles to realize only the "Maternity home" solution so that the "Crematorium" solution would be damped as far as possible. But it depends on the selection of initial phases and the geometry of the system.

Thus, if we use the ideas of the Unified Unitary Quantum Theory appropriately then does not exist a general prohibition for creating of a quantum "perpetuum mobile". Formally there is no such a prohibition even in the general Quantum Mechanics, because there are no conservation laws for a single process under the low energy conditions, but it treats with probabilities instead of this. In other words, the Quantum Mechanics (QM) also offers opportunities for getting energy by collecting of random process someway. It seems that UUQT affords today such an opportunity and suggests the ways how to regulate the values of probabilities.

Together with theoretical investigations plentiful of numerical solutions of equations with oscillating charge were performed, momentum of particles falling with different velocities were summarized and the result was compared to momentum of reflected particles. It was found out that for different repulsive potentials, the total momentum of reflected particles is equal to momentum of the falling particles with a high accuracy, but for a single scattering particle the value of momentum could be either less or more than the momentum of the falling particle. This problem is very complicated and it requires subsequent researches as all this depends on initial conditions (velocity, phase, distance) complexly as well.

The prospects following from the UUQT are not even the most significant. Any flat bans as the impossibility of "perpetuum mobile" creation and any other confirmations of the immovability of conservation laws are unacceptable in philosophy. No, these laws would never be neglected; but there would be such areas in science and technology, very limited in the beginning, so that these laws would be not enough.

The problem of existing of the global conservation laws (we have proved that they are not local laws) is left in abeyance. Nothing but the idleness and atavism of the human thinking lead to it. But this idleness of thinking -concerning the physics-- manifests itself in the intuitive atavism for the Newton laws.

Yes, the conservation laws are incontestable in the classical mechanics and in terms of this theory a continuously operating machine is theoretically impossible. It should be stressed that the conservation laws were transferred to the Quantum Mechanics as an object of worship of the classical mechanics. But the Quantum Mechanics is more fundamental, Newton laws follow from it as a particular case. And if in the terms of the Unitary Quantum Mechanics a possibility to get energy from nothing is theoretically possible, thus a quantum "perpetuum mobile" could be constructed.

It is made possible by means of the equation with oscillating charge. It describes single particles; the difference in their behavior depends on the initial phase of the wave function, but there are no conservation laws for an individual particle at all, they appear only after an ensemble averaging. The equation with an oscillating charge is absolutely new type of motion equation [11-17]. For such equation energy and impulse conversation laws do not exist. It appears after the ensemble averaging. By the way Schrödinger mechanics also do not propose energy conversation laws for small energies (it can offer only a probability of this or that event happening) but it cannot advise how to combine processes and energy liberation while UQT can. A theorem on the circulation does not work in the equation with oscillating charge that allows to use different way to move charge from the point $A$ to the point $B$, but different ways operations will be diverse and this difference should be used.

The authors are trying to design new power plant working at these principles. We think that such a plant will be able to produce energy with extremely small spending of energy. If such power program would be fulfilled on our Planet then it will no doubt result in overheating of the environment. But UQT suggests the solution again: we can construct refrigerating plants which realize the "Crematorium" solution and promote the cooling. Extra heat will disappear. Numerous experiments with the cold nuclear fusion) have shown that nuclear reactions do 
exist but the nuclear reactions products by themselves are not enough for the explanation of huge amount of heat being produced. It is the responsibility of the UQT solutions "Maternity home" $[13-15,19,20,23,34]$. So it looks like catalysis mechanism described [13-15, 22]. Besides all the equation with oscillating charge is quite good in describing the wave properties of the particle. We predict that experiments on the diffraction reflection of electrons from the lattice (classical experiments of Davisson-Germer) can be simulated by supercomputer, but authors do not have such possibility.

Today the science world is agitated by E-Cat of Andrea Rossi that is simply a pressurized ceramic tube with the nickel powder and Hydrogen inside. Under current this tube warms up and generates heat 3-50 times more than consumes. And as we are speaking about Megawatts any manipulation is hardly possible. Few official scientific commissions have concluded that nuclear reactions cannot generate such amount of energy. And even the isotopic composition of Nickel remains stable and heat generation looks absolutely mysterious that does not impede the using of these energy catalyzes.

First that us upset, this statement about that exists the nucleus syntheses at reactions of the $N i$ with hydrogen with formation of $C u$.

$$
N i+p->C u
$$

This is because nucleus syntheses bring about separation of the energy at merging light nucleus. The border lightness serves the nucleus a $\mathrm{Fe}$. The $\mathrm{Ni}$ heavier $\mathrm{Fe}$ already, strictly speaking, metastability and, in principle, capable to nucleus disintegration with separation of the energy than heavier nucleus, that beside it more surplus energy (practically this energy manages to extract only in person events very heavy nucleus $U, P u$ ). Clearly $\mathrm{Ni}$ heavier $\mathrm{Fe}$, therefore for his reaction with proton $\mathrm{Ni}+p=\mathrm{Cu}$ it is necessary to spend the energy! Grains of Nickel (it could be grains or finest crystals) in E-cat have caverns with size of tens Angstroms (they work as potential wells); proton of adequate phase can penetrate inside a cavern. Heat is generated in these caverns under terms of "Maternity Home" as the result of protons numerous knocks on cavern's walls Fig. 6. At present history with E-cat of Andrea Rossi looks the deafening slap in the face to whole modern science [52, 62].

Interestingly enough, there are devices called Testatik Machine M/L Converter from religious group Methernitha. They belong to a religious Christian commune, situated in Linden near Bern. Theirs maker is Swiss physicist Paul Baumann living in the commune. These fantastic devices run as direct current generators, are made as a four dimensions (sizes) type with power value of $0.1,0.3,3$ and $10 \mathrm{~kW}$. In outward appearance this device resembles an electrostatic machine with Leyden jars, so familiar from school physics laboratory. There are two acrylic discs with 36 narrow sectors of thin aluminium stuck to it. The discs rotate in different directions and their mechanical energy is hundreds times lower that produced energy it accounts for about 100 $\mathrm{mW}$ in measurements. The largest device with the power value of $10 \mathrm{~kW}$ has disc diameters more than $2 \mathrm{~m}$, and the smallest has $20 \mathrm{~cm}$; the device with the power value of $3 \mathrm{~kW}$ has $20 \mathrm{~kg}$ in weight. There is no cooling or heating of the air during the long operation of the device, it just smells of ozone there. It was found out that the inventor doesn't clearly understand the principle of operation of the device.

Professor S. Marinov (Austria), whom the commune had given as a present the device with the power value of $100 \mathrm{~W}$ wrote in his book called "Difficult way to the truth --documents on the violation of conservation laws", issued in 1989 by International Publishers East-West: "I can confirm without any doubt that this device is a classical "perpetuum mobile". Without any initial impact, it could rotate an unlimited long period of time and generate electrical energy equal to $100 \mathrm{~W} .$. In that device, the motor and generator are connected... However, it is not clear how it is possible".

The authors of the Unified Unitary Quantum Theory know approximately how this device is constructed, but in this article we are going to do only what is absolutely clear: we are going to show that the operation of this device completely corresponds with the UUQT. Evidently, it operates due to the charge separation concept.

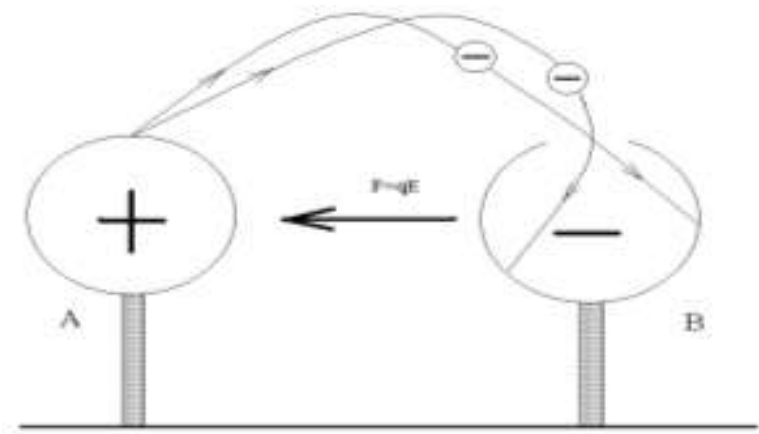

Fig. 7. Work for transferring the charge depends on the mode of transferring and on the path. 
Let us consider two metallic spherical surfaces with a hole isolated from the Earth and from each other. If we carry a first electron from sphere $A$ to the inner surface of sphere $B$ through the hole by means of an isolated stick, then there appears a potential difference. Further, if we carry the second one and the subsequent electrons, sphere $A$ would attract the carried charge, and $B$-would repeal it. It is clear that to move the charge we will have to spend energy. (Fig. 7).

In the Technical University MADI (Moscow) professor V.I. Utchastkin gives lectures on the Unified Unitary Quantum Theory (UUQT) and new energy sources. In his explanations, he uses the figurative analogy: Let us consider a sack of potatoes which mass is $\mathrm{m}$. If we carried it to the fourth floor (the height is $\mathrm{h}$ ), then we spend the quantity of work opposite to the gravitational field which is equal to mgh. And if we throw it down we would get kinetic energy $m v^{2}$, and these quantities would be equal to each other. But we could also carry not the whole sack, but every potato one by one. The work of one quantum of a potato s transfer depends on time, velocity and coordinate, and it must be carried in such way that the spent work would be minimal. If you carry the whole sack in this way, you can get the quantity $m v^{2} / 2>m g$. So, there are no changes in the system, but the energy has appeared.

\section{THE CONSERVATION LAWS AND UNITARY QUANTUM THEORY}

Inventors and swindlers of every stripe and range many years tried to construct or even to design "perpetuum mobile", i.e. imaginary mechanism able to work without outside energy supply. Peter the First (Russian Emperor Peter Great) had even established Russian Academy of Science for such researches (see V.L. Keerpechev, "Talks about mechanics", Gostechisdat, 1951, page 289), but today persons from modern Russian Academy of Science do not like to recollect that circumstance. At the other side French Immortals have decided in 1775 to consider no projects of "perpetuum mobile", and it seems they have not been mistaken jet. However one mistake is known: Daniel Bernoulli was awarded a prize by French Academy for mathematical proof that a boat with engine and screw propeller would never have faster speed than sailing ship!

Magnificent successes of classical thermodynamics have strengthened Humanity confidence in Divine Infallibility of Conservation Laws. Today it is considered nearly indecent to call in question these laws.

First of all let us clarify the origin of conservation laws in classical mechanics [13-15,19,20,23]. Nearly each textbook contains a statement that Energy Conservation Law (ECL) results from homogeneity of time, Momentum Conservation Law results from homogeneity of space, and Angular Momentum Conservation Law - from isotropy of space. And so many people are impressed that Laws themselves result from space-time properties that nowadays are no doubt a relativistic conception. But for example angular momentum is not a relativistic conception already. Therefore such restricted approach is not totally correct, Newton's second law of motion or relativistic dynamics equation and concept of system closeness should be attracted. More over the requested space- time properties themselves are usually wrongly being interpreted. For example, it is assumed that time homogeneity means simple equivalence among all moments of time and homogeneity and isotropy of space means equivalence of all its points and absence of preferential direction in space (all directions are equal) correspondingly.

But these statements are sensu stricto wrong. For example, within many mechanical systems the Earth center direction and horizontal direction differ in principle (for example, pendulum clock located in horizontal plane will not work at all). We can say the same about the body being at the top of the hill, it is able to roll dawn independently, but according to classical mechanics it never climbs by itself. And for a person, being young or old, these moments of time are not equal at all. Hereinafter we would like to explain in what way all that should be understand.

Time homogeneity implies that, if at any two moments of time in two similar closed systems somebody run two similar experiments, their results would not differ.

Space homogeneity and isotropy means that if closed system is moved from one part of the space to another or oriented in other way, nothing would be changed.

Derivation of energy and momentum conservation laws from Newton equation is quite simple in idea. Viz., let us write down the main equation of dynamics in form of

$$
\mathbf{F}=\frac{d \mathbf{P}}{d t}
$$

For closed system $\mathrm{F}=0$ (there are no external forces) and the equation possess the integral

$\mathbf{P}=$ Const

expressing the momentum conservation law.

Now let's write the main equation of dynamics in the form: 
$\mathbf{F}=m \mathbf{a}=m \frac{d \mathbf{v}}{d t}$

and scalar-wise multiply it by $\mathbf{v}$

$\mathbf{F} \cdot \mathbf{v}=m \frac{d \mathbf{v}}{d t} \mathbf{v}=\sum_{i=1}^{3} m \frac{d v_{i}}{d t} v_{i}=\sum_{i=1}^{3} m \frac{d}{d t}\left(\frac{v_{i}^{2}}{2}\right)=\frac{d}{d t}\left(\frac{m v^{2}}{2}\right)$,

where $v$ is a modulus of velocity vector $\mathbf{v}$. For the closed system $\mathbf{F}=0$ it exists the integral

$\frac{m v^{2}}{2}=$ Const

expressing one of the forms of energy conservation law.

Using the definition of the angular momentum for the particle, i.e.

$\mathbf{L}=[\mathbf{r} \times \mathbf{P}]$

and differentiating it both parts by $t$, we obtain

$$
\frac{d \mathbf{L}}{d t}=\left\lceil\frac{d \mathbf{r}}{d t} \times \mathbf{P}\right\rceil+\left\lceil\mathbf{r} \times \frac{d \mathbf{P}}{d t}\right\rceil
$$

As the momentum vector is parallel to velocity vector, the first bracket is equal to zero. And basing on the equation and on definition of central force, as one not creating a momentum, we get

$$
\left\lceil\mathbf{r} \times \frac{d \mathbf{P}}{d t}\right\rceil=0
$$

and

$\mathbf{L}=$ Const.

In the case of central force within unclosed system angular momentum remains constant in value and direction.

The energy and momentum conservation laws can be easily obtained within relativistic dynamics from relativistic relation between energy and momentum

$$
E^{2}=P^{2} c^{2}+m^{2} c^{4}
$$

The term $m^{2} c^{4}$ is an invariant, i.e. it is similar within all reference frames. In other words it is a some kind of constant. This relation can be written in rather different form

$E^{2}-P^{2} c^{2}=$ Const

To satisfy that relation one should admit that

$$
E=\text { Const } \quad \text { and } \quad P=\text { Const }
$$

And that is nothing else than energy and momentum conservation laws.

But strictly speaking there is in relativistic mechanics there is a law of conservation of four-momentum vector $P^{\mu}$, but we are not going to stop at these details.

In accordance with the classical mechanics, the energy conservation law signifies that energy of closed system remains constant, hence, if at the moment $t=0$ the energy of such system is denoted by $E_{0}$, and at the moment $t$ is denoted by $E_{t}$, then

$$
E_{0}=E_{t} \text {. }
$$

In accordance with standard quantum theory, the energy conservation law is laid down in the same way. Within that theory we have the same integrals of motion as in classical mechanics. Some value $L$ would be an integral of motion if

$$
\frac{d \hat{L}}{d t}=\frac{\partial \hat{L}}{\partial t}+\lceil\hat{H}, \hat{L}\rceil=0
$$

As $\lceil\hat{H}, \hat{L}\rceil$ is determined by commutator of operator $\hat{L}$ and of Hamilton's operator $\hat{H}$, so any quantity $L$, 
being not evidently dependent on time will be an integral of motion if its operator commutes with $\hat{H}$. When quantity $L$ is not evidently dependent of time, then the first terms in (3.2.2) vanishes. As remainder we have

$$
\frac{d \hat{L}}{d t}=\lceil\hat{H}, \hat{L}\rceil,
$$

and, as we know, the quantum Poisson bracket vanishes for the integrals of motion being not evidently dependent on time. Thus,

$$
\frac{d}{d t}(L)=0
$$

In any good work dealing with quantum theory it was shown that probability $w$ to observe at any moment $t$ any value of such motion integral $L$, does not depend on time either. We will denote below such integrals of motion $L_{n}$. As far operators $\hat{L}$ and $\hat{H}$ commuted they had common eigen-functions that were functions of stationary states. We should note that the last were obtained from solution of Schrödinger equation without time (not containing $t$ ) which is derived from full Schrödinger equation if

$$
\Psi(\mathbf{r}, t)=\Psi_{0}(\mathbf{r}) \exp \left(i \frac{E}{t}\right),
$$

i.e. if this equation has the periodic solutions. The solutions of Schrödinger equation not containing $t$ satisfy conservation laws, which are, in fact, dictated by condition of total time-independence. The expansions of such solutions in eigen-functions' have the form

$$
\begin{aligned}
& \hat{L} \Psi_{n}=L_{n} \Psi_{n}, \\
& \hat{H} \Psi_{n}=E_{n} \Psi_{n}, \\
& \text { where } \\
& \Psi(x, t)=\sum_{n} c_{n} \Psi_{n}(x) \exp \left(-i \frac{E_{n}}{\hbar} t\right)=\sum_{n} c_{n}(t) \Psi_{n}(x), \\
& c_{n}(t)=c_{n} \exp \left(-i \frac{E_{n}}{\hbar} t\right)=c_{n}(0) \exp \left(-i \frac{E_{n}}{\hbar} t\right) .
\end{aligned}
$$

As (3.2.4) is eigen-functions' expansion of the operator $L_{n}$, the probability does not depend on time, i.e.

$$
w\left(L_{n}, t\right)=\left|c_{n}(t)\right|^{2}=\left|c_{n}(0)\right|^{2}=\text { Const }
$$

We should note once more that it is the probability to observe some given value that is time-independent, while, the value itself is occasional in each individual case. As far the energy is an integral of motion and probability $w(E, t)$ to find out at the moment $t$ energy value to be equal to $E$ is time-independent, then:

$$
\frac{d w(E, t)}{d t}=0
$$

Quantum energy conservation law in the above mentioned form assume the possibility of energy determination at the current moment of time not taking into account its uncontrolled changes due to influence of the process of measurement itself. That situation did not rise any doubts within classical mechanics. But according to quantum theory (as we have written already in [13-15]), the energy can be measured without disturbance of its value only up to

$$
\Delta E \geq \frac{\hbar}{\tau}
$$

where $\tau$ - is the duration of measuring process. Formally, there are no troubles for energy conservation law, as the energy is the integral of motion and we have arbitrary large time interval to accomplish long measuring. For example, let measure within time $\tau$, then leave the system alone for the time $T$, and then measure the energy once again. The energy conservation law in standard quantum mechanics states that the result of the second 
measuring will coincide to $\Delta E \approx \frac{\hbar}{\tau}$ with the results of the first measurement. But even according to standard quantum theory all this is not totally logical, because really existing vacuum fluctuations may meddle and they are able to change the result. Here we have evident violation of conservation law due to vacuum fluctuations, although the integrals of motion exist (contrary to UQT). The standard quantum theory carefully avoids the question of conservation laws for single events at small energies.

Usually that question either does not being discussed at all, or there are said some words that quantum theory does not describe single events at all. But these words are wrong, because the standard quantum theory describes, in fact, single events, but is able to foreseen only the probability of that or other result. It is evident that at that case there are no conservation laws for single events at all. These laws appear only after averaging over a large ensemble of events. As the matter of fact it can be easily shown that classical mechanics is obtained from quantum one after summation over a large number of particles. And for a quite large mass the length of de Broglie wave becomes many times less than body dimensions, and then we cannot talk about any quantum-wave characteristics any more.

It is well known that local laws of energy and momentum conservation for the individual quantum processes are valid within all experiments at high energies only. We cannot say so in the cases of law energies at least due to uncertainty relation and stochastic nature of all predictions in quantum theory. The idea of global but not local energy conservation law is invisibly presenting in quantum mechanics and in any case is not new. From the physical viewpoint it just means that in stationary solutions with fixed discrete energies (standard quantum mechanics) the velocity of a particle reflected from the wall is equal to the velocity of an incident particle. If the particle energy decreases at each reflection, then that case corresponds to solution type "Crematorium" and if increases - to "Maternity home" solution. The scenarios under which events will be developed depend on the initial phase of the wave function and particle energy.

In the strict Unitary Quantum Theory and in the theory of quantum measuring [5, 6] un-removable vacuum fluctuations part a great role. It is quite clear these fluctuations being totally unforeseen and non-invariant with respect to space and time translations. In other words, within UQT there are no habitual space-time properties. Now space-time is heterogeneous and non-isotropic. For example, if the experiment is replaced in any other point of the space or repeated at other time, then in the point where the particle's parameters were examining and particle is interacting with macro-device, another value of vacuum fluctuations would appear (differing from the previous one) that would give another result. Of course that is true for small energies and individual events (particles) only.

The Unitary Quantum Theory is much more destructive with regard to the notion of Closed System. For single events at small energies that notion is inapplicable at all because at any moment of time and in any place where the particle is located (for example, within potential hole) vacuum fluctuation may be abruptly changed. It may occur thanks to various causes; either due to the nature of vacuum fluctuations, or due to the tunneling effect of other random particle.

Sometimes it is stated that energy conservation laws follow from E.Noether theorem, although those results have been contained in the works of D.Gilbert and F.Klein. For any physical system, the motion equations of which can be obtained from variational principle, every one-parameter continuous transformation, that is keeping the variation functional invariant, corresponds only one differential law of conservation and then there exists explicitly conserved quantity. However, it can be easily seen that vacuum fluctuations being imposed on varying functional (Lagrangian) does not remain constant (in any case it seems so today) under parametrical transformations. That consideration does not work too without ensemble averaging either.

In other words, all requirements that lead to classical laws of conservation are absent now. It is hard to expect that the entire laws of conservation will remain valid in that situation for the single particles at small energies. But nowadays it seems that classical laws of energy, momentum and angular momentum conservation for the single quantum objects do not work at small energies due to the periodic appearance and disappearance of particles. All direct experimental checks of the conservation laws were carried out in the cases of great energies but in the cases of small energies for single particles probability results can be obtained only. In that case it is indecently even to recollect the idea of conservation law.

And now a bit of Philosophy for reader. Local Energy Conservation Law (LECL) for individual processes results from the Newton equations for closed systems. It is naive to think that its local formulation will remain constant forever. And it would be a gross error to transfer ECL without alterations from Newton mechanics to quantum processes inside microcosm. Definitely speaking references to the first law of thermodynamics are baseless because it is a postulate. For example, in his letter to one inventor the famous Russian mathematician N.N. Lousin wrote: "First law of thermodynamics was a product of unsuccessful attempts of the humanity to create "perpetuum mobile" and frankly speaking did not follow from anything". Today we can say with more belief that no resourceful machines within the network of Newton mechanics are able to realize "perpetuum 
mobile", and the decree of French Academy, accepted in 1755 to consider no projects of "perpetuum mobile" is still valid. We should add that is apparently true for all projects based on Newton mechanics only.

It is characteristic of the understanding the position ECL in modern physics that this low is bringing down, especially in theory, to the rank of second-order conclusion from the equations of motion. Some physicists reduce ECL to the statement of the first law of thermodynamics, others as for example D.I. Blochintsev [79] consider that "it is quite possible with further development of new theory ECL form will be transformed". As F. Engels wrote in his "Natural dialectics": "...no one of physicists does not, in particular, consider ECL as everlasting and absolute law of the nature, as a law of spontaneous transformation of substance motion forms and quantitative permanency of that motion at its transformations." Many of them are thinking in another manner as, for example, M.P. Bronshtein. He wrote in his work "Substance structure" ECL is one of the basic laws of Newton mechanics. And nevertheless Newton had not attributed to that law rather general character that law had in reality. The reason of that Newton mistaken point of view at ECL was quite interesting... Now it is understandable that in the light of the above mentioned such point of view was not wrong at all. And we should remind that Newton had foreseen in his "theory of bout" many things even quantum mechanics.

At the other side, the founders of quantum mechanics perfectly understood that the conservation law for the single quantum processes at small energies did not exist at all. So, the first thought that understanding of ECL on a par with the second law of thermodynamics, as statistical law, being correct on average and not applicable to the individual processes with small energies, appeared as despair and went back to Erwin Schrödinger first and then to N. Bohr, Kramers, Sleter and G.Gamov. In 1923 Bohr, Kramers and Sleter in despair tried to construct the theory according to which in the process of dispersion energy and momentum conservation laws were satisfied statistically on the average during long time intervals but were inapplicable to the elementary acts. Leo Landau even called that as "Bohr perfect idea".

According to that theory, the process of dispersion should be continuous, but Compton electrons are emitted in a random way. The authors assumed both processes of wave dispersion and Compton electrons dispersion were not connected with each other (?). The main idea was to lay a bridge between quantum theory of the atom and classical emission theory. There were introduced specially so called "virtual" oscillators which generate in accordance with classical theory waves (non quantum one) enable to induce the transition from the state with lower energy to the state with higher energy. These waves did not carry the energy, but power necessary for atom transition from lower to the higher state was generated within the atom itself. Along with that the inverse process of the atom transition from excited state to the lower one could take place, but the energy was not taken away by waves but should disappear inside the atom. In other words, the increase of one atom energy was not connected with energy decrease in another one. Authors considered that these processes compensated each other on average only and that compensation was the better the more events are participated.

Energy conservation law has statistical character according to that interpretation, and there is no law of conservation for single events, but they appear in processes involving large number of particles, i.e. at transition to Newton mechanics. But then it should be acknowledged that in the case of Compton Effect the changes of motion direction of the light quantum and its energy to be appeared in the result of collision were happening apart from the changes of electron's state. The unfounded of such an approach was lately experimentally proved by Bote and Geiger. To say the truth, the authors abandoned that point of view later; moreover at that time this idea did not follow from quantum theory equations. And to get out of the tight spot it was declared that quantum mechanics did not describe single events at all. Thus the most striking paradox was removed by a simple prohibition just to think about it! But genius idea that laws of conservation are not valid for individual processes and appear in quantum mechanics after statistical averaging does not become less genius even if those for whom it "has come to mind" rejected it. May be, this idea was a little premature and should have a somewhat different shape. Contrary to that Unitary Quantum Theory describes single particles. And the alteration of their behavior is determined not only by initial values of its position and velocity but also by initial phase of the wave function (of the wave packet). Then for the single particle local conservation laws do not exist at all. And that is quite another question how to measure the initial phase or any other parameters of a single particle.

Let us examine the following virtual experiment. For more simplicity let use in our reasoning some quantum ball-particle. If classical ball is running to the wall (for simplicity assume it as perpendicular), the velocity of the reflected ball would be equal to its initial velocity (we neglect friction and consider the ball and the walls as totally resilient). In the case of quantum ball the velocity of the reflected ball in various experiments with similar initial circumstances will have the whole spectrum of values: there will be balls reflected with the velocity higher than initial, equal to it and lower then initial. And all these will be described by means of quantum mechanics within uncertainty relation.

Let us ask what would be if we place a second wall parallel to the first one in such a way the ball at each reflection increased its velocity? Then we would get the growth of the ball energy without any efforts from our side. The aim of future constructors of such systems of XXI century would be the necessity to create such initial 
conditions for the great number of particles forming the object, that is realized the sole solution "Maternity home" and is suppressed as far as possible the other solution.

It is evident from the above-mentioned that at competent exploitation of the Unitary Quantum Theory ideas the principle prohibition for "perpetuum mobile" does not exist. Formally as it was shown above that prohibition does not exist even in standard quantum mechanics (there is no laws of conversation for single processes with small energies), and to get energy the particles should be selected in some way (grouping together all random processes with excess energy). But the standard quantum mechanics refuse to describe single events and is not able to advise the way for grouping. As it seems today, the Unitary Quantum Theory gives us such an opportunity.

However, by efforts of scientific groups, interested in their own stability because of simple instinct of selfpreservation the great idea of free energy generation was distorted to such a degree everybody who starts to talk about it is taken for mad.

The modern experimental physics have examined the correctness of conservation laws for huge energies in single cases and for large macro-object when ensemble averaging is used, but the area of small energies is terra incognita.

\section{THE PROSPECTS}

Let us remember the problem about the maintenance of long-term flights to the outer space with electricity. The Prof. Utchastkin's analogy describes precisely a theoretical approach for solving this problem. Of course, there is a great deal to do though, to understand what phenomenon will play the role of those quantum potatoes and how to construct an instrument that would be able to support a minimal energy to bring them to the fourth floor. How can a spaceship be supplied with energy during many months of flight? Near the Earth, photovoltaic cells are used but the more the distance to the Sun is increasing, the more needless they are; using of a nuclear energy source is problematical for different causes.

Today we can neither improve this situation considerably nor do we have even any theoretical conditions which could let us approach it. On the base of such a situation there are common ideas of the construction of matter and its properties. Now then, a new conception of physics is being proposed. Like many others as well. If we stay by the space technology, it s over constructing of engines based on new principles of energy production, maintaining of real-time telecommunication on the distances in outer space, free of limits which are proper to the diffusion of electromagnetic waves. It follows from the foregoing that UUQT opens up a perspective of a solution for the communication problem on extremely wide distances in outer space, excluding the limits of information exchange between Earth and spaceship. The theory also predicts the approaches to creating of the new energy sources and of the new types of engines that would be almost ideal for creating of spaceships of the future.

Conventional jet propulsions transform the conducted energy in the kinetic energy of the beam of a working body flowing from the engine, and the reaction force of this beam the pulling force accelerate the spaceship. Therefore space flights to extremely wide distances will require huge stocks of working body. A classical progression curve reflects the velocity increasing of a thrown-off mass of the working body. Though there is a possibility for creating of a very weak constant pulling but (!) without throwing off of mass.

Let us use the method of analogy again. Regard a classical trick problem in physics for universities admission tests: there is a boat in motionless water and a man with a sandbag in this boat. Can he move the boat by performing any manipulations with the sandbag, for an endless time?

Correct answer: throw the sandbag from the front part of the boat to its back, then carry it back slowly, throw it again and so on. As the viscous friction force by Stocks is proportional to the velocity, the boat will perform swinging motions, over which some linear movement will be applied. Based on this idea, march buggies were constructed in Germany--there is heavy mass moving in there, in one direction quickly and back slowly. Many decades ago, the same effect (Dean`s engine) was wide-ragingly discussed in the USSR in popular science magazines and on TV.

There is a similar phenomenon in the classical electrodynamics as well as in the quantum electrodynamics and it $\mathrm{s}$ related to the Lorentz radiative friction force. The appearance of Lorentz force becomes evident by considering the interaction of the charge and the field caused by it. For a motionless charge the force of such an interaction or self-action is equal to zero, otherwise the free charge would experience a self-acceleration. The charge begins to move, but the electromagnetic field, as its spread velocity is finite, can't reschedule immediately. The accelerated charge practically flies onto its own field; with other words, this effect can be described as appearance of energy flow which is directed upstream to the flow and slowing it down. It generates electromagnetic viscosity which value is related to the acceleration.

How can this phenomenon be used? If there is a charge cloud in flat capacitor, it is possible to make it swing between sheets with different values of acceleration forwards and backwards by applying a sawing motion to the sheets. Because of different forces of radiation friction in the alternate and opposite direction, pulling force 
appears along the lines of electric field. The radiation of such accelerated charges is always perpendicular to their movement and can be screened, but the most important thing on it is the fact that it doesn't change its impulse in relation to the direction of the capacitor s field. It may be paradoxical, but it seems that we get a pulling force by spending energy for this process without throwing-off of any mass in the direction, which is opposite to the motion's one. The authors (L.S.\&V.D.) even published in the US-magazine Journal of New Energy vol.5, \#1, 2000 an article, containing an exact analytical solution of this problem: the pulling of some micrograms appears in a flat capacitor, containing a cloud of $10^{19}$ electrons in which the distance between the sheets is many meters long, by applying of sawing potential of millions of volts. Of course, it is an insignificant result in relation to such a huge (hypothetical) instrument employment, and the using of electron cloud in a flat capacitor has practically no prospects. Curiosity, but similar jet propulsion was created in UK with name Emdrive by Roger Shawyer. But if stabile charged particles exist which mass is at least one billion of electron mass, then this idea becomes very interesting from the technical point of view. Do such stabile charged leptons exist at all and how is it possible to generate them in a sufficiently large number? Today nobody can give an answer..

To generate pulling it is still possible to throw off the mass/ matter, created potential hole, accelerating in it in the same moment. Generally, UUQT allows such solutions that are evident from the "Maternity home" solution. Let us consider the results. UUQT will in future let us solve several basic problems of the worldwide energy supply and all problems in outer space: immediate information changing, the problem of energy supply and constructing of new engine types. It is absolutely precipitant to make technical plans for those solutions, but the foregoing should be considered not as a wanton imagination, but as a possible future program of fundamental researches to transpose our civilization to new physical principles.

The UQT ideas are presented in instinctively absolutely clear picture of quantum events in terms of figures and movements. And philosophical principal of Complementarity can be now retired with well-deserved honors. In spite of mathematical complexity, the UQT delivers the physics from ordinary Quantum Mechanics paradoxes and consequently frank words of Richard Feynman:"I can easily say that nobody understands quantum mechanics" will become the property of history.

Moreover, it became possible:

1) to obtain after solving some QUT equations an electron charge with the high precision;

2) to obtain after solving the scalar telegraph equation the mass spectrum of numerous elementary particles with appropriate precision the mass spectrums of numerous elementary particles[14-16, 18]. The same spectrum was followed from the solutions of the Schrödinger equation and Klein-Gordon integro-differential equations. The risk of computed mass spectrum being random is less than $10^{-60}$. Of course such results cannot be obtained without sacrifice. What would be offered in sacrifice if Ordinary Quantum Mechanics is replaced by the Unitary Unified Quantum Field Theory (UUQFT):

1. There are no strict principles of superposition in UQT. It is violated if wave packets are colliding.

2. There are no strict close systems in UQT and the Conservation Laws work for big energies only. Note that the Conservation Laws forbid beginnings of the Universe.

3. The classical relativistic relation between energy and impulses is valid in UQT only after averaging of observed phenomena and Relativistic Invariance itself is not "the sacred cow".

4. The Space in UQT is not homogenous and not isotropic and has complex geometry.

5. The particles and their interaction are not local.

6. The existing Standard Model Quantum Theory of Elementary Particles requires much alteration.

7. The velocity concept as quotient from division of the traversed path to sometime interval is not quite appropriate in UQT.

If a wave packet (particle) is spreading along the Metagalaxy and then appearing somewhere else, what should we do with the rate, if nothing moves between the points of disappearance and arrival, does it mean that particle has just simply disappeared and then appeared in a new place? There was observed resembling crushing defeat of physics 50 years ago as "weak interaction" burst, so to say, into physics. As soon UQT is nonlinear, it automatically combines all four interactions that can pass from one into another distance. There was observed resembling crushing defeat of physics 50 years ago as "weak interaction" burst, so to say, into physics.

\section{THE LORENTZ TRANSFORMATIONS}

Everything went very well, until the Austrian

General Headquarters interfered: the shells were

taken to the rear, and the wounded to the front.

Jaroslav Hasek,

"The Good Soldier Schweik" 
There is a statement in Special Theory of Relativity that affects the mankind like a sleep-inducing mantraparadox: suppose there are two observers with rules and watches sitting in two objects and moving straight-line and with constant speed in direction to each other. Then from the 1st observer point of view the watch of the 2 nd observer is slow because he is moving. But the 2nd observer can (?) stipulate that he is at rest and the 1st observer' watch is slow. To find out which watch is slow indeed the observers should meet, but that will infringe the terms of inertia - constant and steady motion. The experiment shows the returning watch is slow and this time lag relates to the changes of the gravity potential. But if we return the rules their lengths will not be changed, and that is quite strange because both effects are closely associated.

We would like to show this mantra is absolutely false. Imagine the 1st observer is sitting of the rain drop falling with the constant speed in the terrestrial gravitational field, while the 2nd observer is on the Earth. By this doubtful statement of Special Theory of Relativity the 1st observer can say that his drop is at rest and that the 2nd observer together with the Earth is flying towards him. If observers are not absolute idiots the first observer should ask the second about the source of such a great amount of kinetic energy. This statement can have a little sense only if the masses of the 1st and 2nd objects are equal. The main problem is misunderstanding that any motion is absolute, this idea is thoroughly discusses by the authors in the recent works [53]. It's quite complicated [30, 31].

With other hand the special relativity is in fact Lorentz transformations (1904) derived by V.Vogt (1887) in the century before last. These transformations followed from the properties of Maxwell equations which are also proposed in the nineteenth century (1873). One of these equations connecting electrostatic field divergence and electric charge (Gauss' law of flux), in fact is just another mathematical notation of Coulomb's law for point charges.

But today anybody knows that Coulomb's law is valid for fixed point charges only. It doesn't work for the frequently moving charges. Besides anybody knows that lasers beams are scattered in vacuum one over another, which is absolutely impossible in Maxwell equations. That means that Maxwell equations are approximate and for the moving point charges experimental results essentially differs from the estimated ones in the case charges areas are overlapping.

Few people think about the shocking nonsense of presenting in any course of physics of point charge electric field in the form of a certain sun with field lines symmetrically coming from the point. But electric field is a vector, and what for is it directed? The total sum of such vectors is null, isn`t it?

There are no attempts to talk about, but such idealization is not correct. We should note that Sir Isaac Newton did not use term of a point charge at all, but it s ridiculous to think that such simple idea had not come to him! As for Einstein, he considered "electron is a stranger in electrodynamics". Maxwell equations are not ultimate truth and so we should forget, disavow the common statement about relativist invariance requirement being obligatory permission for any future theory.

To reassure severe critics we should note that UQT is relativistic invariant, it allows to obtain correct correlation between an energy and impulse, mass increases with a rate, as for relativistic invariance just follow of the fact that the envelope of moving packet is quiet in any (including non-inertial) reference systems. To be honest we should note that subwaves the particles consist of are relativistic abnormal, at the same time envelope of our wave packet being immovable in all coordinate-systems corresponds to of Lorentz transformations.

The success of Maxwell equations in description of the prior-quantum view of world was very impressing. Its correlation of the classical mechanics in forms of requirement to correspond Lorentz transformations was perfectly confirmed by the experiments that all these had resulted in unreasoned statement of Maxwell equations being an ultimate truth.

Other reasons for this were later very carefully investigated by a disciple of one of the authors (L.S.), Professor Yu.L.Ratis. (S.Korolev Samara State Aero-Space University), who has formulated the modern spinor quantum electrodynamics from the UQT point of view:

1. Maxwell equations contain constant $c$, which is interpreted as phase velocity of a plane electromagnetic wave in the vacuum.

2. Michelson and Morley have never measured the dependence of the velocity of a plane electromagnetic wave in the vacuum on the reference system velocity as soon plane waves were mathematical abstraction and it was impossible to analyze their properties in the laboratory experiment in principle.

3. Electromagnetic waves cannot exist in vacuum by definition. A spatial domain where an electromagnetic wave is spreading is no longer a vacuum. Once electromagnetic field arises in some spatial region at the same moment, such domain acquires new characteristic, because it became a material media. And such media possesses special material attributes including power and impulse.

4. Since electromagnetic wave while coming through the abstract vacuum (the mathematical vacuum) transforms it in a material media (physical vacuum) it will interact with this media.

5. The result of the electromagnetic wave and physical vacuum interaction are compact wave packets, called photons. 
6. The group velocity of the wave packet (photon) spreading in the media with the normal dispersion is always less its phase velocity.

All abovementioned allows making unambiguous conclusion: the main difficulties of the modern relativistic quantum theory of the field arise from deeply fallacious presuppositions in its base. The reason for this tragic global error was a tripe substitution of ideas--velocity of electromagnetic wave packets 'c' being obtained in numerous experiments physics was adopted as constant 'c' appearing in Maxwell equations and Lorentz transformations. Such blind admiration of Maxwell and Einstein geniuses (authors in no case do not doubt in the genius of these persons) had led XX century physics up a blind alley. The way out was in the necessity of revision of the entire fundamental postulates underlying the modern physics. Exactly that was done by UUQFT [13-15, 19, 23].

Some time ago CERN has conducted repeated experiments of the neutrino velocity measurement that appeared to be higher than velocity of the light. For UUQFT they were like a balm into the wounds. The administration of CERN renounced after sometimes these results considering them as the consequence of experimental errors. As far as the authors know, not all participants of this experiment agree to such renouncing. Besides, many astronomers detect superluminal velocities during observations of stars and galaxies [29]. In fact the movements in excess of the light velocity were discovered earlier by numerous groups of researches. Nearly everybody disbelieved it [29]. The importance of these experiments for UUQFT is settled in the article [23] where at the page 69 it is written that this should be considered as direct experimental proof of UUQFT principle.

There are also other ideas [30, 31]. For example, at «New Relativistic Paradoxes and Open Questions», by Florentin Smarandache, shows several paradoxes, inconsistencies, contradictions, and anomalies in the Theory of Relativity. According to the author, not all physical laws are the same in all inertial reference frames, and he gives several counter-examples. He also supports superluminal speeds, and he considers that the speed of light in vacuum is variable depending on the moving reference frame. The author explains that the red shift and blue shift are not entirely due to the Doppler Effect, but also to the medium composition (i.e. its physical elements, fields, density, heterogeneity, properties, etc.). Professor Smarandache considers that the space is not curved and the light near massive cosmic bodies bends not because of the gravity only as the General Theory of Relativity asserts (Gravitational Lensing), but because of the Medium Lensing.

In order to make the distinction between "clock" and "time", he suggests a first experiment with a different clock type for the GPS clocks, for proving that the resulted dilation and contraction factors are different from those obtained with the cesium atomic clock; and a second experiment with different medium compositions for proving that different degrees of red shifts/blue shifts would result. To regret, the authors today have no decisive position to these complicate questions. Note, this question is terribly complicate and probably is to be leaved to next generations. From one side, the time in UQT exists, so to say, in our head only. From other side, the Lorenz Transformations describe correctly some experimental facts, for example, the mass growing with velocity. Otherwise, all atomic accelerators would be out of order. Thereafter, it is a big mistake to consider all Special Relativity Theory as erroneous. The attitude to the Special Relativity Theory is today highly vague and may be compared in full with the discussion among painters about significance of the Malevich picture "The black square".

Curiosity from the side the Special Relativity Theory declares that the spreading velocity of the information and of the signals cannot exceed the light velocity. At the same time today it is well known that the gravity interaction spreads with the velocity exceeding many times the light velocity. Laplace [42] has obtained corresponding estimates long ago. But this problem is not discussed in any way in Special Relativity.

Over a hundred years passed since the special theory of relativity had been formed. Nowadays it is thought to be absolutely correct, although it was hardly criticized in different countries, and something like medieval inquisition even took place in the USSR and then in the Russian Academy of Sciences in response to the theory. To illustrate the methods of judgment, we cite a paragraph from an article by Academician E. Lifschits published in "Literaturnaya Gazeta", No 24, 1978, where he publicly claimed a paranoiac everyone who dared to criticize the theory of relativity: "I see two types of scientists. Some of them are persons with paranoid psychic deviations... Not swindlers in science but simply not quite normal mentally... They are generally engaged in fundamental problems and deny quantum physics, the theory of relativity etc..."

And all this took place in spite of the fact that by the time this accusation was published Academician E Lifschitz had been well familiar with a large heap of scientific facts proving the absurdity of what he considered "the theory of relativity". He was also well familiar with those methods of organized political violence employed for implementing this "greatest theory" into practice. And there came the result: "... during the year of 1966 only, the department of general and applied physics of RAS USSR helped medical specialists to identify' twenty four paranoiacs " thus entrusting the Academy with the witch-hunting functions for stamping out dissent in physics.

However, numerous honest and courageous scientists do exist in Russia and in the world, for instance. Prof. V. Krasnoyarov, Doctor of Philosophy [38], who wrote as follows: "With all due respect to the scientific 
community, one cannot get rid of the thought that it has been mislead (for non-scientific reasons) and was forced to wear the fool's hat of relativism. We feel painful and humiliated but science must pass a hard path of its purification."

\section{THE SPECIAL THEORY OF RELATIVITY AND UQT}

The absence of alternatives confuses the mind totally.

\section{Henry Kissinger}

The authors must accept it honestly that before the main results concerning the Universal Quantum Theory were generated and published, they had not much doubted the conclusions drawn from the Lorentz transformations. The broad scientific community generally did give a hostile reception to the conclusions about time slowed down in a rapidly moving watch. This has not confused us till today, for the Lorentz transformations can be drawn from the light speed (electromagnetic waves) independence of the speeds of its source or the observer, which seems completely discouraging as far as common sense in concerned, and the slowing down of time and the length contraction of a ruler are simply an elementary consequence of this discouraging fact of experimentation. On the other hand, numerous experiments are performed today [27, 29, 39, 53] that demonstrate speed changing of electromagnetic waves if watched by moving observers and sources but this fact has not been brought up for discussion.

Transformations of coordinates and time were first published by Voigt at the beginning of 1887 completed by Lorentz in 1904 and finally referred to as the Lorentz transformations. Poincare and Einstein, dissatisfied with the fact that the Newtonian mechanics was invariant relative to the Galilean transformations, came to the conclusion (1904-1905) that the equations of mechanics should be changed so as to be invariant relative to Lorentz transformations, which led, in mechanics, to mass growing with velocity. This was experimentally confirmed by Kaufmann (1902-1903). The Maxwell theory united various phenomena, previously dissipated, and the special theory of relativity started its triumphant march around the world.

Nobody was aware in these victorious years of the Coulomb law (the Gauss theorem as one of Maxwell's equations) being only true for charges stationary with respect to each other. Besides, as was experimentally shown later, scattering of electromagnetic waves one on another took place in vacuum and could not be described by Maxwell's equations since they are linear. Nobody approached this problem once again, although it is absolutely clear today that electrodynamics is not a theory of last resort ant it does not seem reasonable to demand that any upcoming theory should be invariant relative to Lorentz transformations.

It should be mentioned that Maxwell's equations were initially written using quaternion formulation [36], the vectors $E$ and $B$ were employed later, but the initial equations contained the total time derivative. The equations were invariant with respect to Galileo's transformations and Lorentz's transformations had not even been planned. Then Hertz and Heaviside [37] introduced the vector and scalar potentials $A$ and giving rise to nonhomogeneous wave equations of second order, which was unknown in Maxwell's ignition formulation, and the total time derivative was replaced by the partial one. These equations were regarded as the final formulation of electrodynamics and are believed to require no changes. They are now considered as relativistically invariant but the invariance with regard to the Galilean transformations disappeared from them.

The theory of special relativity went to even greater lengths, and it was claimed, though for no good reason, that there were no velocities larger than that of light, which allegedly invalidated the causality principle but was completely wrong in fact. The causality principle provides one of the general principles of physics establishing the permissible limits of the influence of physical events on one another; it allows no impact of a given event on all the events that have already occurred ("the cause event precedes the effect event in time" and "the future does not influence the past").

The relativist causality principle is even stronger as it also rules out the mutual influence of the events separated by a space-like interval; the notions of "earlier" or "later" are not absolute for them and they change over with the change of the reference frame. The mutual influence of these events would have been possible only with the frame of reference which includes the object travelling at a speed larger than the speed of light in the vacuum. The well-known opinion that superluminal motion is impossible as far as the relativity theory is concerned proceeds therefore from the relativist causality principle and this opinion can be repudiated.

Humanity forgot that nothing beside the Newtonian equations with some additional allowances for other factors is needed to describe the Solar System. If we take into consideration retarded gravitation potential changes in the space then, as was established by Laplace [42], the propagation rate of these changes will be 70000 as much as the speed of light. There is much evidence and experimentation at present showing speeds many times larger than the speed of light $[27,29,39$, and 53] discussed in the vast literature on the subject. It seems funny that faster-than-light neutrinos were first observed and then abandoned even in CERN (otherwise the relativity theory would have collapsed) under the pretext that the cable with glass fiber was badly attached (!). These studies in CERN involved a lot of researchers and as far as we know not all of them share the same opinion but they keep silent ... as submitted to the discipline. 
Incidentally, faster-than-light neutrinos were observed in the supernova explosions [29] and the neutrinos were detected first and the optic explosion was noticed hours later. The problem of medium (aether), easily eliminated by the special relativity theory, is considered apart from its issues. The authors are not of the opinion that the aether as a medium of some particles does exist, and we believe that this most obscure problem of the present must be settled by the generations to come.

Nonetheless, some reproaching stones must be cast towards the relativity theory and electrodynamics. The Lorentz force docs not proceed from Maxwell's equations but it is introduced to electrodynamics by hand! Besides, according to the apt remark made by Einstein himself that "the electron is a stranger in electrodynamics" and the true equations must not contain point charges or masses. Incidentally, Sir Isaac Newton never applied the concept of a material point and it is naive to imagine that such a simple idea never came into his mind.

One more irregularity concerning Lorentz's transformations seems to occur: they cannot be fully verified, for the moving watch or the ruler needs to be brought back for verification, which contradicts the condition of the inertia property. Experimentation shows that those watches were slow which returned back for they underwent acceleration... It seems curious that in the paradox of the rulers (which is directly connected with time deceleration) the moving ruler does not change its length after coming back... One must agree that this is very strange...

The solving of the Unitary Quantum Theory brought to light, quite unexpectedly for the authors, some consequences from the Lorentz transformations. It appeared that the principal relativistic correlation between energy and impulse was only correct after averaging. According to UQT, the particle-wave packet periodically appears and disappears when moving (gets smeared over the Mega Galaxy). If the particle is spread out it loses its mass and impulse although it retains its energy in the form of harmonic constituents and the relation

$$
E^{2}=p^{2} c^{2}+m^{2} c^{4}
$$

comes out as the averaging.

The growth of particle's mass with its growing velocity is now governed by quite other reasons: when the forcing frequency of the moving particle's appearances and disappearances $\omega_{B}=\frac{n}{7}$ approaches, due to dispersion, the natural frequency of the oscillations of the packet $\omega_{s}=\frac{m}{y}$ and the general resonance with the packet's amplitude growth occurs when $v-$, then mass growth takes place. The standard graph of the dependence of the particle's mass on its speed is now simply half the amplitude-frequency characteristic of the forced oscillations of a harmonic oscillator with no dissipation, and the mass growth is absolute. One may ask us: respective to what medium is the particle moving if you have not yet maintained it till now? Once again we shall honestly answer that we do not know it, and that we do not like the idea of aether. If aether is the medium then we do not understand why its influence is nor expressed either in the laws of motion in the Solar System or in the spectrum of the hydrogen atom and why the motion about it is almost imperceptible.

It seems to us that the gravitation field creates something like the stage or the boards in a theatre where all the processes of the Universe are acted. Time is not accelerated nor decelerated in different reference frames, but the rates of all processes are simply equally changed under the effect of the changing gravitation potential because the mass changes. If an operating watch arrives back it is slow as it have undergone acceleration, which is equivalent to the changing of the gravitational potential. Gravitation and inertia arc one and the same thing and this is one of the most profound physical ideas of the General Theory of Relativity. To elucidate this is the task of the generations to come.

According to UQT, multi-particle production after the collision of high-energy particles (with a large amplitude of the packet) with some periodical structure of another particle is simply the diffraction process of the interaction of non-linear waves one on another, and the jets of the resulting particles are diffraction maxima. The relativity principle is abandoned in UQT but the relativistic correlation (1) takes place in averaging. It appeared, when solving UQT non-linear integro-differential equations, both relativistic and non-relativistic, that in both the evaluation of the permanent fine structure $[21,22,27]$ and the mass spectra calculation of [26, 2830] of many elementary particles - the solution had to be sought for in the inherent system, and time as a parameter tightly connected with space was completely disregarded in the analysis. No fundamental constants, except for and $e$, were made use of.

So time is regarded here as purely Newtonian and it only exists in our mind, and the requirement of relativistic invariance seems to be a hundred-year long illusion of man. The world is not solely electromagnetic waves. Incidentally, UQT have settled up the problem of reversibility: it now does not exist in the Unitary Quantum Theory [29, 30, 37] and the direction of the time arrow is determined by entropy.

The most valuable result of the unitary quantum picture of the world - serious suspicion in validity of Lorentz transformations for every aspect of the world pattern. The World is not only electromagnetic waves, the matter were earlier examined by the authors in their works $[3,4]$ and we are not going to recur to this subject again. 
The main result - four-dimensional relativistic space of time does not exist at all. According Unitary Quantum picture of the world time is Newton number and it is used by our minds for description of dynamic processes only, nature have no idea of time at all and consider the world as complex geometry of space.

The direction of time arrow is determined by entropy. But today the world science is protecting both special and general relativistic theories. Statement "velocity lights this at most possible velocity in nature" is mistake. Any deflection of the light from rectilinear (for example in gravitation field) will be an impossible, since module of velocity after deflection will become more velocities of the light. But today amicable agreement science protects as special, so and general theory of relativity.

Unfortunately, thousands of years of science and philosophy progress have led humanity back to Saint Augustine's words "If nobody asks me I know what time is, hut if I am asked then I am at a loss what to say ".

\section{THE STANDARD MODEL}

As soon relativistic invariance underlies every of the numerous quantum theories of the field, it leaves a devilish imprint at everything. Nevertheless relativistic ratio between energy and impulse although being absolutely correct in fact are not obligatory follow from relativistic invariance only and can result from another mathematical reasons that will be discovered in future. Nowadays Standard Model (SM) contains the most elegant mathematical miracles of researches which hands were tied with relativistic strait-jacket and it not so bad describes these experimental data. Amazing that it was possible to think it out at all.

Nowadays to confirm SM one should find a ""Higgs boson"" and for this purpose the governments of some countries assigned essential sums for the construction of Large Hadrons Collider (LHC). For entire SM the interaction with Higgs field is extremely important, as soon without such a field other particles just will not have mass at all, and that till lead into the theory destruction.

To start with we should note that the Higgs field is material and can be identified with media (aether) as it was in former centuries. But SM authors as well as modern physics have carefully forgotten about it. We would not like to raise here once again the old discussion about it. It $\mathrm{s}$ a quite complicated problem and let us leaves it to the next generation.

But another problem of SM has never mentioned before: in the interaction with Higgs field any particle obtains mass. As for ""Higgs boson"" itself, it is totally falling out of this universal for every particle mechanism of mass generation! And that is not a mere trifle, such mismatching being fundamental fraught with certain consequences for SM.

After "Higgs boson" discovery nothing valuable for the world will happen except an immense banquet. Of course boson will justify the waste of tens billions of Euros. But even now some opinions in CERN are expressed that probably boson non-disclosure will reveal a series of new breath-taking prospects and where were these voices before construction, we wonder? But that's not the point! If this elusive particle were the only weakness of SM!

To our regret today this theory cannot compute correctly the masses of elementary particles including the mass of ""Higgs boson"". More worse, that SM contains from 20 to 60 adjusting arbitrary! - parameters (there are different versions of SM). SM does not have theoretically proved algorithm for spectrum mass computation and no ideas how to do it! With other hand in SM no place for dark matter, but in UKT there is [58].

All these bear strong resemblance to the situation with Ptolemaic models of Solar system before appearance of Kepler`s laws and Newton s mechanics. These earth-centered models of the planets movement in Solar system had required at first introduction of so called epicycles specially selected for the coordination of theoretical forecasts and observations. Its description of planets positions was quite good; but later to increase the forecasts accuracy it had required another bunch of additional epicycles. Good mathematicians know that epicycles are in fact analogues of Fourier coefficients in moment decomposition in accordance with Kepler`s laws; so by adding epicycles the accuracy of the Ptolemaic model can be increased too. However that does not mean that the Ptolemaic model is adequately describing the reality. Quite the contrary.

Note the following remarkable fact: the standard theory allowed to detect spectra by using always the quantum equations with outer potential and as corollaries to geometric relations between de Broglie wave's length and characteristic dimension of potential function. The quantum equation of our theory does not contain the outer potential and describe a particle in empty free space; the mass quantization arises owing to the delicate balance of dispersion and non-linearity which provides the stability of some wave packets number. It is the first case when spectra are detected by using the quantum equations without outer potential. 
“...the kernels are pure emeralds, but people, it may be, lie..."

\title{
XV. THE NUCLEAR PHYSICS
}

\author{
A.S. Pushkin
}

Nuclear physics as a part of quantum theory is very luckless. Thus the potential of the strong interactions is so complicated that no one even very bulky and intricate mathematical expression is able to describe with more or less veracity the experiments of two nucleons interaction. This interaction depends in very complicated manner from all parameters of the nucleons movement and their orientation towards vectors of velocity, acceleration, spin, magnetic movement, etc. Scarcely one can find a parameter which practice interaction does not depend on. From UQT point of view the strong interactions appear in the result of nucleons represented by the wave packets overlapping. Today the way of mathematical notation of the overlapping wave packets interaction is absolutely vague as soon nonlinear interaction in any space-time point of the waves is different due to different amplitudes.

It's a really complicated problem as soon there is only one nonlinear mathematical problem existing for each space-time point and even with the intuitive clearance of situation we do not expects its soon solution. The complete understanding of the nuclear structure hardly can be expected in the soonest time without exact expression for the potential of the strong interaction.

In general it should be noted that quantum world looks more clear and simple in UQT than in the general quantum mechanics, but we cannot repeat it while speaking about the mathematics used. The appearance of the exact analytical solution of the scalar problem of elementary particles mass spectrum can be considered as Fate gift (or God's help) for UQT. By the way the standard Schrödinger quantum mechanics has the same gift -- the exact analytical solution of the Hydrogen atoms equation.

The nuclear process at small energies should be reviewed. Today the strict nuclear physics does not assume nuclear reactions at small energies and that contradict experimental data $[54,55]$. Here we should also note our skepticism towards the idea of nuclear fusion in Tokomaks, we consider this way as hopeless. To justify these experiments we have to mention that the solution was obtained in the deficient of other ideas and under the great pressure of the future power problems. But the use of the reactions of classical cold fusion for the power output is also difficult due to the complexity of colliding nuclei phasing $[32,33]$. This phenomenon is well described by the equation with oscillating charge, while the cold nuclear fusion had been predicted in UQT 6 years before its real discovery [7, 11, 15-20, 54-56].

It was discovered long ago that nuclear transmutations are widely spread (it is especially evident for plants and biological objects), but they are faintly connected with energy liberation. The examples of such reactions are:

$$
\begin{aligned}
& \mathrm{Mn}^{55}+p \rightarrow \mathrm{Fe}^{56} \\
& \mathrm{Al}^{27}+p \rightarrow \mathrm{Si}^{28} \\
& P^{31}+p \rightarrow S^{32} \\
& K^{39}+p \rightarrow \mathrm{Ca}^{40}
\end{aligned}
$$

In reactions of such a type very slow proton (its kinetic energy is equal practically to zero) is penetrating inside the nucleus by the above-mentioned way and stays there. There is no nuclear energy liberation, because the nucleus remains stable both before and after reaction. In accordance with classical nuclear physics, the nucleus, as usual, after a charged proton with great kinetic energy gets inside it, becomes unstable and breaks to pieces, and its fragments obtain bigger kinetic energy. The reactions of above-mentioned type were considered impossible at all at small energies and therefore were not studied in the classical nuclear physics. Apparently, that is absolutely new type of nuclear transmutations unacknowledged by modern nuclear science, but experimentally discovered sufficiently long ago. Today there are a lot of experimental data confirming the mass character of nuclear transmutation [32, 33, 54-56]. Moreover there are many projects of nuclear waste neutralization that use this method.

\section{THE SOLID-STATE PHYSICS}

The band theory of solid is based at the point on the solution of the problem of an electron movement in the field of two or more charges. But this problem does not have analytical solution yet, in practice a speculative quality solution is used only. The results are that electrons in the solid have quite specific allowed power bands. This field of the science is very successful and hardly will be revised. Any solution of the equations with the oscillating charge for the electron moving in the field of few nuclei also result in appearance of allowed and forbidden bands [14, 15, 21].

Somewhat apart is classical tunneling effect. In UQT the probability of tunneling effect appearance depends on 
the phase of the wave function (in contrast to the ordinary quantum theory, where at the squaring of the wave function module its dependence on the wave phase totally disappears). It could be interesting to prove such dependence by the experiments. It can be easily done if creating a new transistor on the basis of absolutely new principle of the electron current control [21].

We are not going to analyze the modern theory of superconductivity, but we are sure that the equation with oscillating charge will deepen on both understanding of superconductivity as well as mysterious properties of quantum liquids.

\section{THE ASTROPHYSICS AND COSMOLOGY}

The authors regret not being in sympathized with the ideas of the Universe origin from one singular point. The most amazing in this theory is a detailed computation of events occurred in the fractions of the first second just after the Big Bang. Today when the fundamental physics is making only first shy steps towards the real understanding of the quantum processes we still do not have clear model of the particles, or understanding of a spin appearance, of a charge and magnetic moments. At the same time, in Internet were the sensational results obtained inwell-known Lawrence Livermore National Laboratory USA were newly announced. In this Laboratory the space model of our entire Universe after astronomical observations during many years and their analysis using Supercomputers was constructed. It was turned out that our Universe has the flat structure and all Galaxies have dimensions near a half of million light-years being six milliard light-years apart and all Galaxies lie on the same plane (!). Obviously, such picture of our Universe has no relation with the Big Bang model.

According to UQT the processes of the multiple particle production at collision is a common result of the waves packets of big amplitudes diffraction in periodic structures one another, as for the multiple outgoing in different directions particles they correspond to the general diffraction maximums. But we do not assume the responsibility of the mechanism of the multiple particles production for the Universe appearance. To our opinion the complete understanding of the quantum world will arise only after solving of 32 nonlinear integrodifferential equations of UQT [8, 13-15]. To their regret the authors are not able to solve these equations.

And many cosmologists would like to use theories assuming existence Universe localities where the energy is coming into being and also other localities where the energy annihilates. For example, British astronomer Fred Hoyle has developed the theory of Universe where it takes the place the continuous creation of matter. He wrote: "Different atoms constituting the matter do not exist at some given moment of time and then after instant they exist already. I must admit this idea may look as strange. But all our ideas about creation are strange. According to previous theories the whole quantity of matter in Universe was coming into being just as whole and all process of creation looks as super-gigantic instant explosion. As for me, such idea seems much stranger, than idea of continuous creation". (F.Hoyle, La nature de 1 Universe, 1952.)

The official astronomical science does not accept the ideas of F.Hoyle and of some other astronomers (H.Bondi, T.Gold, and P.Jordan) about continuous creation of matter in Universe because the Conservation Laws are considered as infallible. But from the viewpoint of our UQT these ideas are quite not strange.

Our real world continuum consists of an enormous quantity of particles moving with different velocities. Partial waves of the postulated vanishing particles create real vacuum fluctuations that change in a very random way. Certain particles randomly appear in such a system, owing to the harmonic component energy of other vanished particles. The number of such "dependant particles" changes, though; they suddenly appear and vanish forever, as the probability of their reappearance is negligibly small, and so we do expect that all particles are indebted to each other for their existence. Yet, if some particles are disappearing within an object, other particles are arising at the same moment in that object due to the contribution of those vanishing particles harmonic components and vice versa.

The simultaneous presence of all of the particles within one discrete macroscopic object is unreal. Some constituent particles vanish within the object while others appear. In general, a mass object is extant overall, but is not instantaneously substantive and merely a false image. It is clear that the number of particles according to such a theory is inconstant and all their ongoing processes are random, and their probability analysis will remain always on the agenda of future research.

In accordance with UQT there are another solutions for the quantum harmonic oscillator besides stationary, where the given tiny incipient fluctuation is growing, gaining power and finally becoming a particle. It is so called "Maternity Home" solution. There are also other solutions where substance (power) is disappearing. Such solutions have been called "Crematorium". May be Metagalaxy is simply entangled in searching the balance, isn`t it?

All this allows expecting that space continuum in the centers of Galaxies produces different particles, electrons, protons, neutrons, which are the sources of light atoms. Later thanks to the gravitation light atoms are transformed into gas nebulas where under gravity compression the stars are lighting. It s quite possible that the current theory of Stars evolution is correct in general while describing (via Supernova) the production of other 
atoms apart Hydrogen and Carbon the planets consist of. We do not think nuclear process at small energies (which are possible in UQT, but impossible in standard quantum theory) will essentially modify evolutionary view of the Galaxies development.

It is interesting that the state with the minimum quantum values $L=0, m=0$ belongs to the very heavy neutral scalar particle (WIMP) with our name Dzhan and mass about 69.6 TeV, which in principle should purely interact with the others $[15,16,18,56,58]$. With the growth of the quantum numbers the mass of the particle is diminishing. So there should be a lot of Dzhan-particles due to the small quantum numbers. And probably their existence is responsible for the dark matter in general, in accordance with some evaluations Metagalaxy consist of up to $80-90 \%$ of the dark matter.

\section{THE GRAVITATIONAL THEORY}

Hell is empty and all the devils are here.

"The Tempest" William Shakespeare

It seems Gravitational theory should follow from 32 nonlinear integro-differential equations of UQT and the authors are expecting that it can be done in future [8, 13-15]. Nevertheless we will make now some conservative assertions. The current data regarding the Universe expansion can be interpreted as the change of the gravitational potential sign (gravity is replacing by repulsion) at great distances for the great masses. Probably the difference between absolute the values of electric charge of a proton and an electron, say in 15-20 signs, is responsible for his phenomena, but for us this idea is extremely unsympathetic.

Gravitational interaction remains an extraordinary mysterious appearance in UQT as actually it has a very high speed of interactions distribution and approximately is in times weaker than electro-magnetic interactions. The origin of such an enormously big number remains the greatest riddle.

On the other hand if any particle is a package of partial waves of some uniform field, probably is possible a following curious phenomenon which was observed and described by us more than once earlier [13-15, 19, 23]. If to put a ditch with the substance having abnormal dispersion on a way of the wave package moving in flat Euclidean space, the package after ditches can appear even if it is situated at distance of many light years from a package as formally mathematically harmonious components exist on all infinite rectilinear coordinate of package movement as ahead of it, and behind. Thus the package can disappear in that place where it was, and to appear at huge distances ahead of a package, or behind. Thus the package didn't move at all between points of disappearance and new appearance, and the normal idea of speed in the unitary quantum theory loses its initial meaning.

Similar teleportation was observed of ten times. Probably, it is actually a long-range action, (couple longue distance) observed in gravitation. A curious though appears that the waves building a package, could be connected with gravitation and all particles consists of a gravitational field. Then this field can be a stage or a scene where all other processes with final speeds of interaction transfer are played. It will allow connecting the quantum theory and the gravitation theory which while aren't connected yet today in the future. But it is a task for the future generations.

At the same time according to the processed information (Hlistunov at all [28]) from Russian Command-andMeasuring Complex for the monitoring and control of the space objects at the entire moment of collision comet Shumejker-Levi with Jupiter geodesic satellites "Tope-Poseidon" and "GEO_IK" began swaying at their orbits. Normally the orbit of a geodesic satellite lies inside the tube with about $1 \mathrm{~km}$ diameter and the orbit can be control with the high accuracy not more than one meter precision for the position data and centimeters per second for velocity. During the collision the sensors registered 5-8 times increase of the trajectory tube diameter. In the same article Hlistunov [28] at all on the basis of correlation analysis of the position data measurements and information obtained from earthquake-detection station it was shown that the waves of gravitational potential variation were the trigger for earthquakes. With other hand official science in Russia did not know about it [57].To the authors regret they do not have the similar information from NASA.

\section{THE GENERAL THEORY OF RELATIVITY AND UQT}

The situation in GTR (the gravitation theory) is even more scandalous. The Authors do not regard themselves as the coryphées in the fields of Riemann's geometry and tensor analysis; nevertheless they are quite confident that GTR by all means bears most profound ideas of physics that will undoubtedly retain in the future theory of gravitation. But, in fact, the conception of the dependence of space properties on the distribution and motion of masses was for the first time put forward and developed by Jacobi in ... 1848. Then this conception was further expanded in the works of a whole plead of such physicists as Lipke, Berwald, Frank, Eizerhard [38, 40, 41]. Nowadays we understand that the spectrum of masses $[16,18]$ and the fine structure constant $[9,10]$ owe their appearance only to geometry and to the properties of space.

The fact that any motion is regarded as absolute in UQT is highly positive for this theory, as was for the first time noted by Academician A.D. Alexandrov [45] at the All-Union Conference "Space and Time in Modern 
Physics" in 1959. He said that "our issue is particularly about a mathematical theorem and, therefore, the statement that the theory is based on "the general relativity principle " (whose senselessness was admitted by Einstein as far back as in 1916) is equal to someone's allegation that "the Einstein theory relies on the general law according to which $2 \times 2=5$... Therefore, GTR rather does eliminate the relativity of motion than extends it from inertia I motions to any accelerated ones " [45].

Still many leading scientists, both in Russia and abroad, definitely deny GTR at all. The President of the American Physical Society and the Nobel Prize Winner Prof. E.Wigner stated as a well-approved fact [50] that "such fundamental physical concepts as a coordinate and an impulse, which might be assigned any random initial values, do not bear any physical sense within the frame of GTR".

Vice-President of the Russian Academy of Scientists Acad. A.A.Logunov [46-48] proves that no physical sense is borne by such fundamental physical value as mass within the frame of GTR. Moreover, he wrote unambiguously [49] that "the energy-impulse tensor in the Einstein theory - has the same relation to physics as does the last-year snow to the mystery of the Tunguska Event". When speaking to the UNESCO session in March 1986, Acad. A.Logunov suggested that some special international agreement should be created for expelling GRT from research as one having nothing to do with natural sciences. His article in a magazine ("Tekhnika Molodezhi", No 10, 1986) carries his opinion that "the energy-impulse vector is always equal to zero in GRT and GRT no concept of energy can be found there".

Theory will be entirely useless if not supported by appropriate experimentation. As regards the quantum science, theory and experiment in it show coincidence with an accuracy of 6 to 9 significant figures. Unfortunately, GRT cannot boast such coincidence. We shall briefly analyze main direct experimental confirmations of the theory. Three of those are the most important. The other ones can be liable to another classical interpretation.

1.The deviation of a star beam in the Sun's gravitational field during solar eclipse. GRT predicts a 1.75" deviation of the stellar beam whereas the Newtonian theory stands for a value two times as small. The Sun has an immense plasma cloud over its surface, which also deflects the light and this deflection is tens of times larger than the predicted effect is. The plasma cloud's parameters are unknown and surely similar predictions are made to achieve needed results. The same considerations work when quasar radio emissions in the Sun's field are measured.

2. Expansion of the Universe according to the Hubble law. The Hubble constant has changed by orders of magnitude since the observations started but all the time it corresponds to the theoretical predictions (!).

3. The motion of the perihelion of Mercury. It has been for long known in observational astronomy that owing to other planets' gravitation Mercury's motion is not simply elliptic but the planet travels along an ellipse that rotates for 575" every hundred years. Corrections based on the Newtonian theory make it to be 532". The remaining value 43 " cannot be interpreted within the frame of the Newtonian theory.

Not exactly... It takes the Sun about 30 days to make a full rotation on its axis.... That is why it is a bit oblate (like the Earth)... Then the Sun's gravitational field will rely on the angle (with no spherical symmetry), and Mercury's trajectory will certainly make a turn... We do not insist that this deviation will be 43 " but it will of course exist. To solve the problem correctly, one needs to know what the Sun's polar and equatorial radius, which have never been measured and no one knows the way to measure them... Everybody keeps silent about this fact for 43" is considered to be excellently accounted for in terms of GRT...

Not long ago the situation grew absolutely scandalous... The collection of articles "Unsolved Problems in Special and General Relativity " (Chief Editor Florentin Smarandach, USA ) might be referred to as a requiem for the Special and General Relativity theories. The authors are an American, a Russian, the rest are the Chinese. All of them cannot be called engaged persons. The first article of the Collection, "Einstein's Explanation of Perihelion Motion of Mercury", is by Chinese mathematician Hua Di [31, page 5]. The author pointed to a rude mistake made by Einstein when calculating the error of 43" by way of integration, and the result must have been not 43" but 71.5". We were so astonished that rushed to make sure whether it was so. Sad to say this, but we all had the same result $71.5^{\prime \prime}$. And what did surprise us mostly was the fact that not only Einstein but the authors of many articles and books had stupidly reproduced these calculations, challenging us to think seriously about the situation just like Prof. Krasnoyarov [38].

To draw a final line in the discussion about the experimental substantiation of the General Relativity Theory (GRT), let us cite the conclusion of French scientist L.Brillouin [44] who left to us his unambiguous estimation: "The conclusion is that no experimental facts exist that would confirm the mathematically cumbersome theory by Einstein. Everything done after Einstein provides mathematically complicated generalizations, additions or modifications not supported by experimentation. Science fiction in the area of cosmology is, frankly speaking, a very interesting but hypothetical thing. "

The above-laid considerations reflect a completely dismal general physical picture of the world. If this picture is further accepted in the scientific community, then many countries will continue wasting their time and money in empty projects like the International Reactor for Thermonuclear Synthesis, Large Hadrons Collider and the like. 
The now existing army of "brothers talc-tellers" will depict for us more and more fantastic physical scenarios. Amazed people will listen to these breathtaking stories about parallel universes, worm holes, the teleportation of large objects, travelling in time, horizontal events and any other stuff like this, and demand more and more money from their Governments for putting up new shows. Leaders of states must remember that "the viability of any idea is determined by the quantity of people feeding on it".

\section{THE CHEMICAL CATALYSIS}

The process of chemical catalysis and the catalysts are the great mystery of the modern science. The number of chemical catalysis theories equals the number of chemical catalytic processes. A specialist in chemical catalysis used to think that this or that reaction is not going because of the needed catalyst has not been found. Even Michael Faraday studied these problems. He seems to say about platinum as being the universal catalyst. Only this (while platinum practically does not react with anything) immediately suggests an idea that chemical processes are not enabled at all and we should look for the physical universal mechanism of reactions.

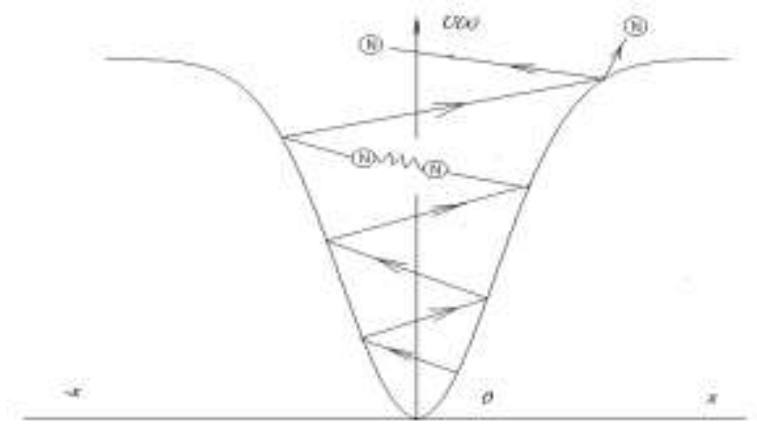

Fig.7. Oscillation of Nitrogen molecule in potential well of catalyst.

We cannot exclude that idea of energy generation within a potential well is just waiting for the creation of general theory of catalysis. Here we should recall brilliant words of a famous Russian specialist on physical chemistry Professor A.N. Kharin (Russia, Taganrog, 1954) who always said at his lectures: "The problem of chemical catalysis is the most incomprehensible in the modern physical chemistry and it won't be solved until physicist discover some new mechanism able to explain the liberation of the energy that lowers the reaction barrier."

Absolutely the same scenario we have for Nitrogen in production of Ammonia. At standard terms molecular of Nitrogen is nearly inert. It can react with Hydrogen only in atomic state. But transformation of molecular Nitrogen into atomic require a lot of energy. It looks like: molecular of Nitrogen penetrates into catalyst cavern and it terms of "Maternity Home" two free atoms of Nitrogen leave cavern and immediately joint protons (Fig.7).

We are sure that in such a way water can be decomposed for Oxygen and Hydrogen. At normal conditions the mixture of Oxygen and Hydrogen is stable. In other words two stable substances (water and gas mixture) are simply divided by a high energy barrier, that can be overcome (tunneling effect analogue) by using the exact catalyst and the UQT ideas. For today a lot of experiments of water decompositions are known, the energy evolved in the process of hydrogen combustion is ten times higher than necessary for decomposition. It makes possible to construct a water-engine for autos.

There are amazing results $[10,11]$ on catalytic decomposition

$H_{2} S=H_{2} \uparrow+S \uparrow$

with separation of the heat and catalytic reaction

$\mathrm{H}_{2}+\mathrm{S}=\mathrm{H}_{2} \mathrm{~S}$

also with separation of the heat! These reactions do not require any additional energy. In laboratory Oak Ridge opening by Adam Rondinone catalyst (fullerene with copper) transforming coal acid (soda water) in ethyl alcohol! But from the point of chemical thermodynamics that is evident infringement of the Energy Conservation Law! According to modern conception no catalyst adds any energy to the catalyzed process. But practice shows that it does! The catalyst adds energy to the process. And the only rational explanation of this fact gives new solutions for quantum oscillator in UQT. This example is not singular in the chemistry of catalysis. Specialists of catalysis are used to deal with excess heat generation; nevertheless they are "normally" ignoring this fact to avoid reputation of "ignoramus" in simple thermodynamic calculations. The role of 
catalyst in modern chemistry of catalysis should be revised. And that was done in [6,9]. Our UQT allows, as we hope, to make the first shy steps in right direction

\section{CONCLUSION}

In essence, our theory discovered new world properties and new theoretical possibility of the radical transformation of the civilization. Let us to remind of the prophetical words of the famous US science-fiction author Arthur Clarke: "Something that is theoretically possible will be achieved practically independent of technical difficulties. It's enough to desire it." (back translation)- Profiles of the Future, 1963.

In conclusion we would like to quote extremely acute words of Louis de Broglie: "Those who say that new interpretation is not necessary I would like to note that new interpretation may have more deep roots and such theory in the long run will be able to explain wave-particle dualism, but that explanation will not be received either from abstract formalism, modern nowadays, or from vague notion of supplementary. But I think that the highest aim of the science is always to understand. The history of the science shows if any time somebody succeeded in deeper understanding of physical phenomena class, new phenomena and applications appeared. Hope that many researchers will study that enthralling question casting aside preconceived opinions and not overestimating the importance of mathematical formalism, whatever beautiful and essential it was, because that may result in loss of deep physical sense of phenomena" (Louis de Broglie, Compt. Rend, 258, 6345, 1964 back translation).

We would like to add the amazing phrase of A. de Saint-Exupéry: "The truth is not something that could be proved, but something that makes all things easy and clear" (back translation).

\section{ACKNOWLEDGEMENTS}

The authors thank for discussions to professors V.A. Boichenko, A.S. Bogomolov, V.V. Graboshnikov, S.I. Konstantinov, A.A. Kostin, M.A. Mokulsky, V.I. Utchastkin.

\section{REFERENCES}

[1] Jammer M. (1961). Concepts of mass in classical and modern physics. Harvard University Press.

[2] Sapogin L.G. (1973). «United Field and Quantum Mechanics», System Researches (Physical Researches) Acad. Science USSR, Vladivostok, 2, pp. 54-84, (Russian).

[3] Sapogin L.G. (1979). «On Unitary Quantum Mechanics». Nuovo Cimento, vol. 53A, No 2.

[4] Sapogin L.G. (1980). "A Unitary Quantum Field Theory". Annales de la Fondation Louis de Broglie, vol.5, No 4, p.285-300.

[5] Sapogin L.G. (1982). "A Statistical Theory of Measurements in Unitary Quantum Mechanics”. Nuovo Cimento, vol.70B, No.1, p.80.

[6] Sapogin L.G. (1982). "A Statistical Theory of the Detector in Unitary Quantum Mechanics".

[7] Nuovo Cimento, vol.71B, No.3, p.246.

[8] Sapogin L.G. (1983), Journal «Technics for a young», No.1, page 41. (Russian).

[9] Sapogin L.G., Boichenko V.A., (1984). "On the Equation of the Unitary Quantum Theory" . Annales de la Fondation Louis d Broglie, vol. 9, No.3, p.221.

[10] Sapogin L.G., Boichenko V.A. (1988). "On the Solution of One Non-linear Equation” . Nuovo Cimento, vol.102B, No 4, p.433.

[11] Sapogin L.G., Boichenko V.A. (1991). "On the Charge and Mass of Particles in Unitary Quantum Theory" . Nuovo Cimento, vol.104A, No 10, p.1483.

[12] Sapogin, L.G., I.V.Kulikov (1995) "Cold Nuclear Fusion in the Unitary Quantum Theory", Chinese Journal of Nuclear Physics, vol.17, No 4, p.360-370.

[13] Sapogin, L.G. (1996) "Cold Nuclear Fusion and Energy Generation Processes in Terms of the Schrödinger Equation" . Chinese Journal of Nuclear Physics vol.19,\#2, p.115-120, 1996.

[14] Sapogin L.G., Ryabov Yu.A., Utchastkin V.I. (2003). Unitary Quantum Theory and a New Energy Sources. Ed. MADI, Moscow, (Russian).

[15] Sapogin L.G., Ryabov Yu.A, Boichenko V.A. (2005). Unitary Quantum Theory and a New Sources of Energy, Archer Enterprises, Geneva, NY, USA.

[16] Sapogin L.G., Ryabov Yu. A., Boichenko V. A. (2008). Unitary Quantum Theory and a New Sources of Energy, Ed. Science-Press, Moscow, (Russian, transl. from English).

[17] Sapogin L.G., Ryabov Yu. A. (2008). «On the mass spectrum of elementary particles in Unitary Quantum Theory», The Old and New Concepts of Physics, Vol. 5, No 3 www.uni.lodz.pl/concepts

[18] Sapogin L.G., Dzhanibekov V.A.(2008), Journal «Technics for a young», No.9,11, February (Russian).

[19] Sapogin L.G., Ryabov Yu. A. (2010). «New Theoretical Results about the Mass Spectrum of Elementary Particles». Applied Physics Research, vol. 2, No 1, p.86-98, May. www.ccsenet.org/apr

[20] Sapogin L.G.(2010), About Unitary Quantum Field Theory Applied Physics Research, vol. 2, No 2, p.114-140, November www.ccsenet.org/apr

[21] Sapogin L.G., Ryabov Yu. A. (2011). "Approximation Equations with oscillating charge in Unitary Quantum Theory and its applications to the analysis of some quantum problems."

[22] International Journal of Applied Science and Technology, Vol.1, No 5, September.www.ijastnet.com

[23] Sapogin L.G., Dzhanibekov V.A., Sapogin V.G. (2011). "A new approach to control electron current in Unitary Quantum Theory" International Journal of Applied Science and Technology, Vol.1, No.6, November. www.ijastnet.com Sapogin L.G., Ryabov Yu. A. (2011). "Unitary Quantum Theory and Catalytic Process Theory".

[25] International Journal of Pure and Applied Sciences and Technolodgy 3(2), pp.93-120 www.1jop

[27] Frontier Research,vol.11, Issue 4,Version 1.0, July. 
[28] Sapogin L.G. (2012), Journal «Technics for a young», No.2, page 2-11, February (Russian).

[29] Sapogin L.G., Ryabov Yu. A.,Dzhanibekov V.A. (2012),"Problems in the Unitary Quantum View of the World" International Journal of Applied Science and Technology,Vol.2, No .5, May. www.ijastnet.com

[30] Sapogin L.G.,Dzhanibekov V.A. (2012),"Object Lessons of the Unitary Quantum Theory "

[31] Journal of Modern Physics and Applied ,Vol., No .1 page 1-22, http://scik.org

[32] Wang L.J. etc. (2000). "Gain-assisted superluminal light propagation", Nature, 406, p.277-279.

[33] W.Hlistunov, Poduvalcev V.V., Mogilyuk J.G. (2011) Science and Education pub. Electronic scientific and technical periodic \#11,November.

[34] Tsao Chang, (2013) "Neutrinos as Superluminal Particles" Journal of Modern Physics, 4, 6-11. Smarandach Florentin (2012) "New Relativistic Paradoxes and Open Questions", Somipress.

[35] Smarandach Florentin-Chief Editor (2013)"Unsolved Problems in Special and General Relativity" Education Publishing\& Journal of Matter Regularity (Beijing) ISBN:9781599732206.

[36] Sapogin L.G., Ryabov Yu. A. (2013) "Low Energy Nuclear Reactions (LENR) - and Nuclear Transmutations at Unitary Quantum Theory", International Journal of Physics and Astronomy, Vol. 1 No. 1.

[37] Sapogin L.G., Ryabov Yu. A.,Dzhanibekov V.A.(2014) "Nuclear Transmutations and Low Energy Nuclear Reactions at the Unitary Quantum Theory" Frontier Research Global Journal of Science Vol. 14 Issue 1 Version 1.0.

[38] Josef Gruber (2008) Raumenenergie-Technik Michaels Verlag.(in Germany)

[39] Sapogin L.G., Ryabov Yu. A., (2014)"Solution of the particles scattering problems in UQT by using the oscillating charge equation“"International Journal of Applied Science and Technology, vol.4, \#4.

[40] Maxwell J.K. "Treatise on Electricity and Magnetism". М.:Наука, 1992.

[41] Heaviside O. "Electromagnetic Theory. Electrical Papers." Vol.1, 2. London- N.Y., 1892.

[42] Бровко Ю.П. “Политика в науке - или наука орудие политики?” Журнал “Природа и Человек. Свет” №7/2002.(Russian)

[43] Marinov St.," The velocity of light is direction dependent" Czech. J. Phys. 1974. B24. N9. p.965-970.

[44] Хайдаров К.А. "Невидимая Вселенная". - BRI, Алматы, 2005.(Russian)

[45] Хайдаров К.А. "Температура эфира и красные смещения". - BRI, Алматы, 2005.(Russian)

[46] Laplace P.С. Изложение системы мира. Т.1, 2. СПб, 1861 .(Russian)

[47] Бронштэн В. "Беседы о космосе и гипотезах". М. Наука, 1968г.(Russian)

[48] Brillouin L." Relativity Reexamined". М. Мир. 1972г. с.83

[49] Alexandrov А.Д. Доклады Всесоюзной Конференции "Пространство и время в современной физике", 1959г. Москва (Russian)

[50] Logunov A. «Новая теория пространства-времени и гравитации». Препринт П-0139. Институт ядерных исследований АН CCCP. 1981Г. .Russian)

[51] Logunov A.А. "К работам Анри Пуанкаре О динамике электрона».М.:МГУ, 1988.

[52] Logunov A.A. "Лекции по теории относительности и гравитации. Современный анализ проблемы". М.:Наука, 1987.(Russian)

[53] Logunov А., Денисов В.. "Инертная масса, определенная в ОТО, не имеет физического смысла". АН СССР. Журнал теоретической и математической физики. т. 51, № 2/82г.

[54] Wigner E. "Symmetries and Reflections" M., "Mir". 1971г.(Russian)

[55] Startsev A.N.,at all.(2013) "Low Temperature Catalytic Decomposition of Hydrogen Sulfide into Hydrogen and Diatomic Gaseous Sulfur". Topics in Catalysis, 56, 969-980. http://dx.doi.org/10.1007/s11244-013-0061-y

[56] L.G. Sapogin,V.A. Dzhanibekov, Y.A. Ryabov (2016) "Enigmatic E-Cat of Andrea Rossi and theUnitary Quantum Theory" Open Access Library Journal, 3: 2833.http://dx.doi.org/10.4236/oalib.1102833

[57] L.G. Sapogin et all (2015) "About the Conflicts between the Unitary Quantum Theory and the Special and General Relativity Theories" Journal of Modern Physics, 4, 6-11. http://dx.doi.org/10.4236/jmp.2015.66083.

[58] Kuznetsov V.D. at all "Low Energy Transmutation of Atomic Nuclei of Chemical Elements", Annales Fondation Louis de Broglie, Volume 28, \# 2, 2003.

[59] Urutskoev L.I. at all Annales de la Fondation Louis de Broglie, Vol. 27, \# 4, 2002.

[60] L.G. Sapogin, Yu.A. Ryabov and V.A. Boichenko, (2015) "The Unitary Quantum Theory and a New Sources of Energy". Science Publishing Group, USA.http://www.sciencepublishinggroup.com

[61] Fortov V. E. at all (1996) Uspehi Physicheskih Nauk vol.166, \#4, p.391 (Russian).

[62] L.G. Sapogin, Yu. A. Ryabov (2015) "Calculation of the Theoretical Mass Spectrum ofElementary Particles in Unitary Quantum Theory" International Journal of High Energy Physics Special Issue: Symmetries in Relativity, Quantum Theory and Unified Theories. Vol. 2 No(4-1): 71-79; doi: 10.11648/j.ijhep.s.2015020401.16

[63] Leo G. Sapogin. The Unitary Unified Quantum Field Theory. International Journal of High Energy Physics. Special Issue: Symmetries in Relativity, Quantum Theory and Unified Theories. Vol. 2, No. 4-1, 2015, pp. 8-32. doi: 10.11648/j.ijhep.s.2015020401.12

[64] Leo G. Sapogin, V. A. Dzhanibekov, Yu. A. Ryabov. "The General Unitary Quantum Picture of the World”. International Journal of High Energy Physics. Special Issue: Symmetries in Relativity, Quantum Theory and Unified Theories. Vol. 2, No. 4-1, 2015, pp. 33-53.doi: $10.11648 /$ j.ijhep.s.2015020401.13

[65] Leo G. Sapogin, Victor A. Boichenko. "Fundamental Equation, Commutation Relations and Relativistic, Invariance at Unitary Quantum Theory". International Journal of High Energy Physics. Special Issue: Symmetries in Relativity, Quantum Theory and Unified Theories.Vol. 2, No. 4-1, 2015, pp. 59-70. doi: 10.11648/j.ijhep.s.2015020401.15

[66] Leo G. Sapogin, V.A. Dzhanibekov, A.A. Kostin, M. A. Mokulsky, Yu.A. Ryabov, V.I.Utchastkin (2017). "Is Unitary Quantum Theory able to change civilization?" Advances in Social Sciences Research Journal, 4(3) 181-189. URL:http://dx.doi.org/10.14738/assrj.43.2714

[67] Humphrey J. Maris, "On the Fission of Elementary Particles and the Evidence for the Fractional Electrons in Liquid Helium", Journal of Low Temperature Physics vol.120, page 173, 2000.

[68] Journal "New Scientist", Marcus Chown, “Double or Quit”, October 14, \# 2260, 2000. 